\title{
De la ville bleue à la métropole grise
}

Fondation, protection et destruction de Kökeqota (Huhehaote)

From the Blue City to the Grey Metropolis. Foundation, protection and destruction of Kökeqota (Huhehaote)

\section{Isabelle Charleux}

\section{(2) OpenEdition Journals}

\section{Édition électronique}

URL : https://journals.openedition.org/emscat/439

DOI : 10.4000/emscat.439

ISSN : 2101-0013

\section{Éditeur}

Centre d'Etudes Mongoles \& Sibériennes / École Pratique des Hautes Études

\section{Édition imprimée}

Date de publication : 1 décembre 2004

ISBN : 2-9518888-2-1

ISSN : 0766-5075

Référence électronique

Isabelle Charleux, «De la ville bleue à la métropole grise », Études mongoles et sibériennes,

centrasiatiques et tibétaines [En ligne], 35 | 2004, mis en ligne le 09 mars 2009, consulté le 13 juillet

2021. URL : http://journals.openedition.org/emscat/439 ; DOI : https://doi.org/10.4000/emscat.439

Ce document a été généré automatiquement le 13 juillet 2021.

(c) Tous droits réservés 


\title{
De la ville bleue à la métropole grise
}

\author{
Fondation, protection et destruction de Kökeqota (Huhehaote) \\ From the Blue City to the Grey Metropolis. Foundation, protection and \\ destruction of Kökeqota (Huhehaote)
}

Isabelle Charleux

1 Kökeqota ${ }^{1}$, la "Ville bleue ", capitale de la Mongolie méridionale ou région autonome de Mongolie-Intérieure (RAMI) en Chine, conservait encore dans les années 1990 un patrimoine architectural d'une grande richesse. C'est ainsi qu'elle mérita en 1987 son classement parmi les "villes célèbres historiques et culturelles de niveau national " de Chine. En 2002, plus de soixante sites ${ }^{2}$ de la municipalité de Kökeqota (qui s'étend jusqu'à une cinquantaine de kilomètres autour de la ville) sont inscrits sur les listes du patrimoine, dont six au niveau national et dix-neuf au niveau régional. Mais face à une forte expansion démographique, économique et urbanistique, le patrimoine architectural, même "protégé", subit des dégradations irréversibles: la vieille ville, hormis ses temples, est en train d'être rasée.

2 Ayant pu presque chaque année suivre l'évolution du paysage urbain de Kökeqota de 1993 à 2002, je voudrais ici dresser un constat permettant de mieux évaluer l'ampleur des dégâts et l'urgence d'une politique de préservation, et aussi de comprendre les enjeux actuels. Il ne s'agit pas de porter des jugements hâtifs sur la politique municipale en matière d'urbanisme ou d'accuser globalement la politique patrimoniale de la Chine. La plupart des pays d'Asie - pour ne s'en tenir qu'à l'Asie - font face à des problèmes similaires. Cependant, en Chine peut-être plus qu'ailleurs, la notion de patrimoine est exploitée à des fins politiques, idéologiques et économiques. Et lorsque le patrimoine concerné est celui d'une des nombreuses " ethnies minoritaires", comme les Mongols ou les Tibétains, il peut devenir le fer de lance de la "politique des minorités", qui consiste à promouvoir et à "folkloriser" un vernis de culture identitaire en surface, pour masquer la destruction, en profondeur, de leur civilisation et de leur religion. 


\section{Histoire de la fondation de Kökeqota}

3 L'histoire de la ville de Kökeqota et une description de son schéma urbain ancien permettent de voir comment cette ville sino-mongole a traversé les siècles, et en particulier le $\mathrm{xx}^{\mathrm{e}}$. Kökeqota est située dans une plaine bordée au nord et au sud de belles montagnes qui la protègent des vents de la steppe. À quelque quatre cents kilomètres à l'ouest de Pékin, la ville apparaît aux voyageurs de la fin du XIX siècle comme une oasis de verdure. Son climat est privilégié par rapport au reste de la Mongolie, et les nombreux affluents du fleuve Jaune, au sud, permettent l'irrigation des terres ; c'est pourquoi la plaine de Kökeqota était nommée en mongol et en chinois "la plaine fertile". Du fait de sa situation stratégique, la région a une longue histoire frontalière faite d'affrontements et d'échanges ${ }^{3}$. Elle fut occupée selon les époques tantôt par des populations d'origine non chinoise - notamment les Xiongnu, les Tuoba (dynastie des Wei du Nord), les Kitan (dynastie Liao), et enfin les Mongols -, tantôt par l'empire chinois (sous les Han et les Tang). Depuis le $\mathrm{IV}^{\mathrm{e}}$ siècle avant notre ère, des portions de la "grande muraille" ont été édifiées dans la région, et l'on recense à proximité plus de 80 vestiges de villes fortifiées: Yunzhong (des Royaumes Combattants aux Han); Shengle (Wei) ; Zhimin zhou (Tang); Fengzhou (Liao), etc.

Kökeqota fut la première ville construite par les Mongols après la chute de l'empire en 1368. Son fondateur est le prince Altan Qan (1507-1582) des Tümed, principal acteur et initiateur de la conversion des Mongols au bouddhisme tibétain de l'école guélougpa (dge lugs pa). Peu après avoir signé les accords de paix de 1571 avec la Chine des Ming, mettant ainsi fin à un demi-siècle de conflits frontaliers, Altan Qan entreprend l'édification d'un palais puis d'une capitale en 1572, qu'il nomme "la Ville bleue", Kökeqota ${ }^{4}$. Les Ming donnent à la nouvelle petite ville le "titre" de Guihua cheng, "Ville revenue à la civilisation". Pour les Mongols Tümed comme pour les Chinois, Kökeqota est le symbole de la paix entre les deux peuples qui y cohabitent depuis sa fondation. Ce sont d'ailleurs des artisans chinois, établis de gré ou de force au nord de la muraille, qui réalisent la construction de la ville et de ses temples. En 1579-1580, Altan Qan fait construire au sud de la ville un premier grand monastère, aujourd'hui connu sous le nom de Jeke zuu, "Grand temple". Proche de la Grande Muraille et des marchés chinois avec lesquels les Mongols font beaucoup de commerce, la ville princière devient rapidement une importante cité marchande, redistribuant les marchandises chinoises vers les autres tribus mongoles. Elle est également, dès sa fondation, une ville sainte, rassemblant trois des plus anciens monastères bouddhiques de Mongolie, et attirant religieux célèbres, traducteurs, missionnaires mongols et tibétains, ainsi que des ermites qui s'isolent dans les montagnes avoisinantes. Au début du XVII e siècle, Kökeqota est nommée dans des sources chinoises Zhaocheng, “ la ville des temples".

5 Pillée par Ligdan Qan des Caqar en 1632 lors de sa fuite vers la région du Koukounor, Kökeqota se relève de ses cendres. Rebâtie et agrandie, elle devient pour les Mandchous une base militaire de première importance, d'abord pour la conquête de la Chine, plus tard, pour les guerres contre les Mongols occidentaux Djoungars. En 1735, l'empereur Qianlong (reg. 1736-1796) ordonne la construction de Suiyuan à trois kilomètres à l'est de Kökeqota. Suiyuan est une garnison et une cité administrative sino-mandchoue, habitée uniquement par les "familles des bannières" - organisation militaire et administrative mandchoue. Suiyuan, dite aussi la "Nouvelle ville" (Xincheng), est 
achevée en 1739 - et, depuis cette date, l'ancienne Kökeqota est couramment appelée en chinois la Vieille ville (Jiucheng). Lorsqu'une partie des fonctions administratives sera transférée dans la Nouvelle ville, religion et commerce seront les activités essentielles de l'ancienne cité.

6 À partir du milieu du xix siècle, l'immigration chinoise va s'intensifier, de sorte qu'à la fin du XIXe siècle les deux villes compteront environ vingt mille habitants, mongols et chinois, en nombre à peu près égal. Les musulmans chinois (Hui) s'y installent aussi pour fonder ou reprendre des entreprises commerciales. En 1880 est créé un office de colonisation, mais c'est surtout à l'arrivée du chemin de fer depuis Pékin en 1919 que la colonisation chinoise, ainsi que la mise en culture des terres autour de la ville, vont croître à un rythme exponentiel.

\section{Aspect physique de Kökeqota sous les Qing (1644-1911)}

$7 \quad$ La petite cité princière d'Altan Qan et de ses descendants comprenait palais, greniers et arsenal, et ne couvrait que $0,1 \mathrm{~km}^{2}$ à l'intérieur d'une enceinte fortifiée, avec des murs de cinq mètres de haut, un fossé et deux portes au nord et au sud. Puis elle s'agrandit vers le sud. En 1694-1696, face à la menace djoungare, Chinois et Mongols, moines et laïcs travaillent ensemble pour réparer l'ancienne enceinte et édifier une muraille autour de cette deuxième ville d'une superficie de $0,2 \mathrm{~km}^{2}$. La ville est alors composée de l'ancienne forteresse, devenue le yamen ${ }^{5}$ du gouverneur mandchou ${ }^{6}$, et de son extension méridionale dont l'enceinte rectangulaire est percée de portes au sud, à l'est et à l'ouest. Un grand axe nord-sud bordé de monastères part de la forteresse et traverse la ville en direction du sud : c'est la Grande rue sud (Danan jie).

8 La densité des édifices religieux intra-muros est impressionnante. Les grands monastères mongols dominent le paysage de la ville. Ces monastères bénéficièrent du patronage des empereurs mandchous jusqu'à la fin du xvIII siècle, mais les subsides impériaux se tarirent au siècle suivant si bien qu'ils connurent de sérieuses difficultés financières. Au début du $\mathrm{xx}^{\mathrm{e}}$ siècle, Kökeqota abrite onze monastères mongols intramuros dont sept grands complexes de plusieurs centaines de moines chacun, et vingthuit extra-muros. On compte alors douze lamas réincarnés ayant le rang de qutuүtu, près de quatre mille moines ordonnés et de nombreux novices. En plus de ces monastères, Kökeqota renferme également vingt temples chinois (six temples du dieu guerrier Guandi ${ }^{7}$, deux temples du dieu de la Littérature, un temple du dieu de la ville - ou dieu des Murs et des Fossés, Chenghuang miao -, deux temples du Roi Dragon, un temple du dieu des Richesses, un temple de Guanyin, etc.), cinq mosquées pour les nombreux musulmans Hui et les caravaniers d'Asie centrale (la première érigée en 1789), une église catholique (au nord de la Vieille ville, près du temple du dieu de la ville) et cinq temples protestants. Ce large éventail d'édifices religieux reflète le caractère multiculturel de la ville.

9 La Nouvelle ville de Suiyuan a un aspect très différent. Cette ville garnison construite selon un plan carré de $6,5 \mathrm{~km}$ de périmètre, avec des murs de dix mètres de haut, présente les caractéristiques d'une ville chinoise modèle, symétrique et ordonnée. Elle comprend des bâtiments administratifs, des maisons de fonctionnaires et des temples dédiés aux divinités chinoises adoptées par les familles des bannières (dieu des 
chevaux, Guandi, dieu de la ville). On comptait également trois temples protestants et une église catholique (juste en dehors de la porte ouest), dont l'existence est encore attestée en 1949 et qui ont été détruits depuis. Les deux villes sont donc bien séparées physiquement et spirituellement, puisque chacune a un temple du dieu de la ville (FIG. 1).

FIG. 1 - Plan de Kökeqota au début du Xx $x^{\mathrm{e}}$ siècle

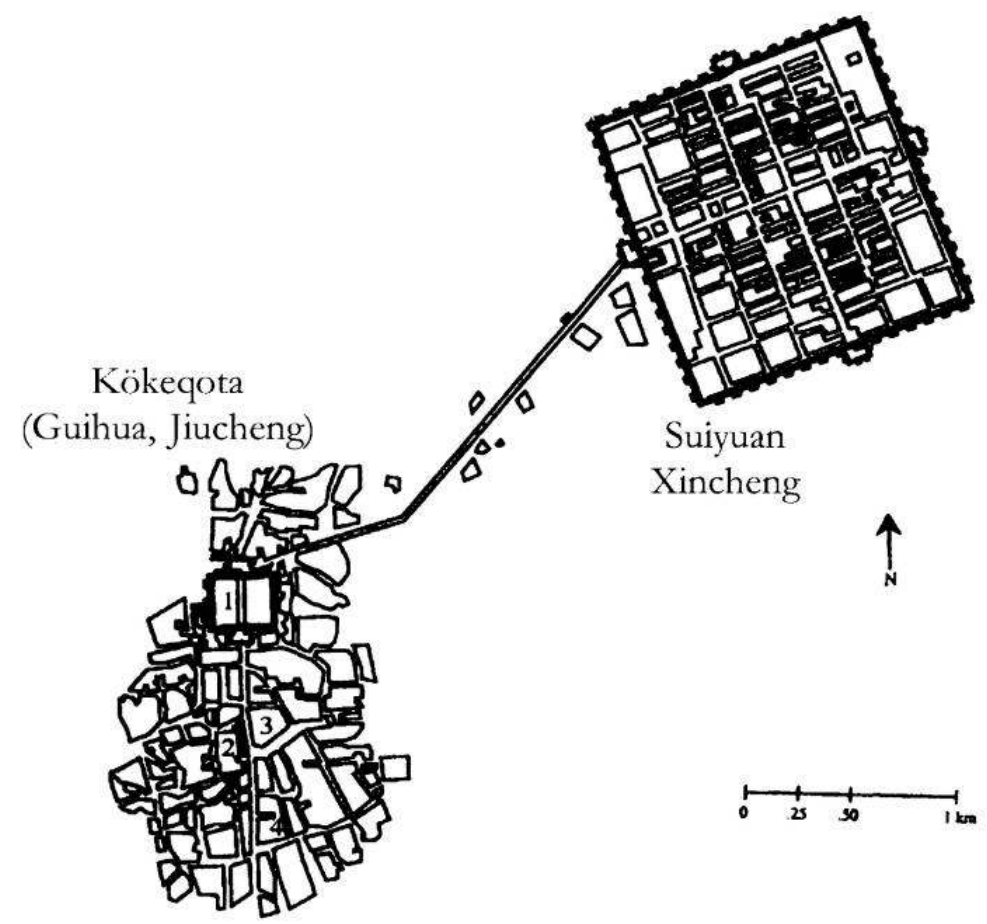

1) Forteresse de Kökeqota ; 2) Jeke zuu ; 3) Siregetü zuu ; 4) Tabun subury-a

d'après Gaubatz 1996, p. 384

Les voyageurs occidentaux et chinois qui visitent la Vieille ville de la fin du XVII siècle au début $\mathrm{du} \mathrm{xx}^{\mathrm{e}}$ siècle la décrivent comme un bourg aux maisons de terre, dont émergent les somptueux monastères ${ }^{8}$. La saleté, l'insalubrité et la pauvreté des maisons et des boutiques pressées sans ordre les unes contre les autres contrastent avec la richesse des bâtiments monastiques. Chinois et Mongols y vivent réellement côte à côte. Les moines mongols sont nombreux à habiter dans les monastères mêmes. Les riches Mongols se font construire par des artisans chinois de belles maisons pour le prestige, mais préfèrent généralement à la maison la yourte dans la cour - ou, plus probablement, vivent dans la maison l'été (lorsque les pluies trempent la tente de feutre), et dans la yourte l'hiver. Certains construisent devant leur yourte une porte élaborée de style chinois avec un petit toit de tuiles et des doubles battants. On trouve également des maisons rondes en briques crues, sur le modèle d'une yourte 9 .

11 Dans la Vieille ville, le yamen, les monastères, les résidences des fonctionnaires, les autres bâtiments administratifs, les nombreux commerces (thé, textiles, peaux de mouton) et les entreprises de transport commercial ne laissent que peu de place aux habitations, d'où une importante extension extra-muros. Les boutiques sont nombreuses ; chaque rue est affectée à une corporation, comme dans les villes 
chinoises. Certaines corporations rassemblent des Chinois d'une même origine géographique, d'autres sont des guildes commerciales mongoles, han et hui (il y avait 33 guildes au début du $\mathrm{xx}^{\mathrm{e}}$ siècle). Elles sont parfois logées dans des temples chinois, comme les corporations des tailleurs, des fabricants de papier et des fabricants d'objets en laine.

12 À partir du milieu du XIX siècle, les terrains et les édifices intra- et extra-muros, qui appartiennent en grande partie aux monastères, sont loués aux commerçants : c'est un phénomène qui se généralise en Chine à la fin de l'époque impériale et sous la République ;les petits temples urbains vivent essentiellement de ces loyers. Un quartier musulman se forme au nord de la Vieille ville et s'étend vers l'est en direction de Suiyuan. Depuis l'ère Tongzhi (1862-1875), une nouvelle muraille extérieure, d'un périmètre de quinze kilomètres, entoure les deux villes.

\section{De la République chinoise à la république populaire de Chine (1911-1990)}

$13 \mathrm{Au}$ début $\mathrm{du} \mathrm{xx}^{\mathrm{e}}$ siècle, la Vieille ville surpeuplée, insalubre et anarchique, fait triste figure. Les habitants prennent les briques des murailles pour construire leurs maisons, augmentant la densité de bâtiments intra-muros. Mais c'est une agglomération pleine de vie. Des aménagements urbanistiques communs à toutes les villes de la jeune république de Chine sont réalisés : la tour de la Cloche est rasée en 1919, la muraille en 1922, à l'exception de la porte nord, finalement détruite en 1958 - mais la Vieille ville n'en est pas pour autant défigurée. En effet, une ville moderne se développe entre le cœur historique de Kökeqota et Suiyuan, sans vraiment toucher aux rues anciennes. C'est dans cet espace nouveau que s'installent les administrations (police, poste) et les écoles. Artisanat, commerce et industrie s'y développent : échoppes où l'on travaille le cuir ou le métal, usine de briques, petite usine de charbon, moulin à farine, quelques fabriques de laine et des centrales électriques, quelques entreprises commerciales pour la production de fer, d'alcool, de sauce de soja. Progressivement, les deux villes jadis occupées par des Mongols, des Chinois, des Hui et des familles des bannières mandchoues deviennent une seule grande ville d'ethnie majoritairement han.

La croissance rapide de Kökeqota va se poursuivre après 1949 pour répondre à la nécessité de doter d'une capitale moderne la région autonome de MongolieIntérieure ${ }^{10}$. Kökeqota passe d'une population de 110000 habitants en 1949 à 523000 (et à plus d'un million pour la municipalité) dans les années 1980, et d'une superficie construite de $1407000 \mathrm{~m}^{2}$ en 1949 à $12670000 \mathrm{~m}^{2}$ dans les années 1980. L'industrie de la laine devient l'activité économique principale, mais on compte également des aciéries, des usines métallurgiques, chimiques, pharmaceutiques, des usines produisant des machines agricoles, des centrales électriques, et des entreprises d'électronique.

Malgré la forte hausse de la population han, la proportion de Mongols est désormais plus élevée qu'en 1949, car beaucoup de Mongols ont été forcés de s'y installer dans les années 1950. En 1956, les Mongols représentaient $6 \%$ de la population totale (14 907 habitants sur un total de 268 211). En 1982, ils représenteraient $13 \%$ des habitants de la Nouvelle ville, $6 \%$ de ceux de la Ville ancienne et globalement $11 \%$ de la population de la municipalité. Ces Mongols, pour la plupart, ne sont pas des Tümed, mais des Mongols d'autres bannières, récemment installés ; on estime que seulement $20 \%$ d'entre eux 
parlent mongol ${ }^{11}$. Ils vivent, éparpillés dans toute la ville, dans les blocs d'appartements associés aux usines. Les Hui se sont également dispersés, mais beaucoup continuent d'habiter l'enclave au nord de la Vieille ville, rebaptisée District du peuple Hui (Huiminqu) et comprenant encore sa vieille mosquée.

Le nouveau quartier entre les deux villes poursuit son développement autour de deux axes majeurs nouvellement créés : l'avenue Sun Yat-sen, Ouest et Est, reliant les deux villes et l'avenue Sili-jin youl, Nord et Sud, partant de la gare. Ces deux directions orthogonales déterminent un quadrillage du secteur en gros blocs d'immeubles séparés par de larges rues (à l'exception de la rue diagonale Zhongshan). Ce quartier comprend les centres culturels et administratifs de la ville (Maison des sciences, siège du gouvernement municipal, siège de la RAMI et bureaux annexes, musée de MongolieIntérieure) et des districts industriels au sud et à l'est. On y a aménagé une grande place de la Chine nouvelle (Xinhua guangchang), à mi-chemin entre la Vieille ville et la ville mandchoue, et le parc du Peuple (Renmin gongyuan), sur l'ancienne colline du Dragon couché ${ }^{12}$. En 1965, près de $5000 \mathrm{~m}^{2}$ d'habitations traditionnelles sur cour sont détruits autour de la gare ferroviaire, et remplacés par des immeubles résidentiels. Près du Grand magasin des Minorités, le siège d'une ancienne société de bienfaisance ${ }^{13}$ fondée en 1928 est l'un des rares édifices anciens préservés dans ce quartier ; il servait en 1998 d'école et de lieu d'exposition de calligraphies.

L'ancienne garnison de Suiyuan a été progressivement démolie. La muraille est abattue entre 1954 et 1957. Des portions entières de Suiyuan sont rasées ${ }^{14}$; on perce de larges artères bordées par des immeubles en béton, des usines et des bâtiments administratifs. Dans toute la ville, on assiste de 1950 jusqu'aux années 1980 à un développement rapide de constructions standardisées destinées à l'industrie et au logement. Les quartiers de la (nouvelle) Ville nouvelle ${ }^{15}$ adoptent le style modulaire, sur le modèle soviétique - modules rectangulaires uniformes, en béton, assemblés autour d'une cour centrale ou d'un parking et ceints d'un mur. Rapides à construire, bon marché, ces immeubles modulaires combinant habitat et lieu de travail sont un symbole du socialisme, moderne et efficace.

Pour les nouveaux monuments publics, les architectes inventent un "style mongol" utilisant la forme de la yourte. On trouve ainsi des bâtiments en forme de yourte géante, tel le Hall d'exposition de la culture mongole (1957), et des édifices surmontés de dômes ornés de trois feuilles de trèfles renversées - motif traditionnel mongol -, tel le bâtiment de l'hippodrome de Mongolie-Intérieure (Nei Menggu saima chang) surmonté de cinq grands dômes (1959) ${ }^{16}$. Le musée de Mongolie-Intérieure (Nei Menggu bowuguan) adopte quant à lui le style "salle-de-bain" (recouvert de carrelages) monumental et est couronné d'une statue de cheval au galop (FIG. 2) ${ }^{17}$. 
FIG. 2 - Musée de Mongolie-Intérieure surmonté d'un cheval au galop

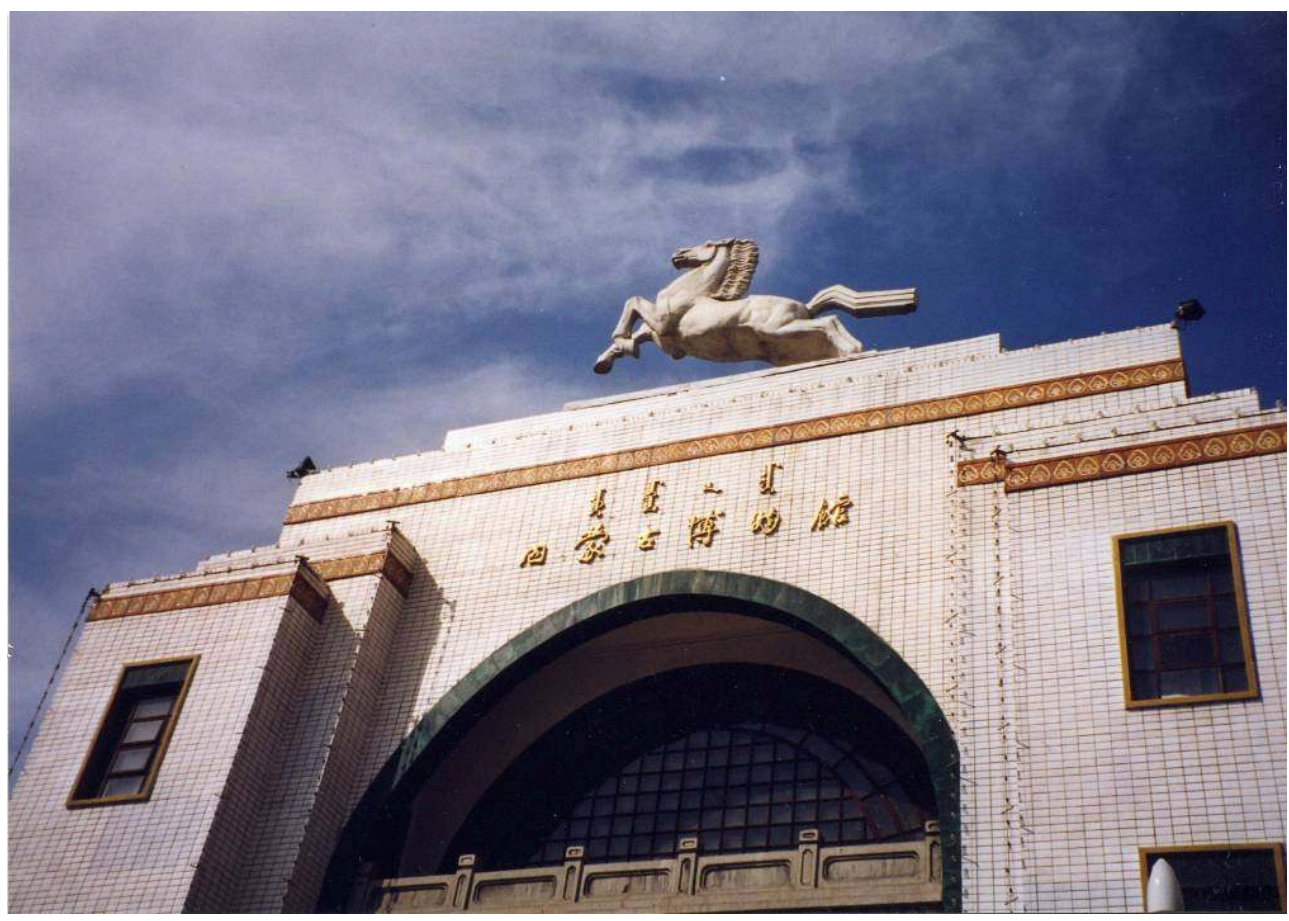

Isabelle Charleux, 1999

19 Face à cette explosion urbanistique, la Vieille ville n'a subi que des aménagements somme toute mineurs, et les destructions concernent essentiellement les temples. L'axe nord-sud est élargi, et la porte nord est démantelée entre 1958 et 1960 pour faciliter le trafic. De nombreux temples sont réaffectés; ils tombent peu à peu en ruine et sont finalement rasés pendant la révolution culturelle (1966-1976). Les noms de rue conservent encore la mémoire des édifices détruits: Jiucheng beimen qichezhan (station de bus de la porte nord de la Vieille ville), rue du temple de Guanyin, rue du Wen miao (temple de Confucius). La persistance de ces anciens noms montre que la muraille et les grands bâtiments servent encore de point de repères. Une grande partie de la Vieille ville a cependant survécu, parce que ses bâtiments étaient encore fonctionnels, qu'il restait de la place ailleurs et qu'il n'était pas possible de tout raser d'un coup.

\section{Les monuments religieux de Kökeqota : protection, restauration, réouverture}

À la veille de la révolution de 1911, quatorze monastères tibéto-mongols étaient encore en activité dans la Vieille ville et aux alentours.

21 À l'époque républicaine, nombreux seront les petits monastères de Kökeqota, en situation économique fragile, à être abandonnés, détruits ou réduits comme peau de chagrin, contraints de vendre leurs bâtiments les uns après les autres; et pourtant, ils ont été relativement épargnés par la politique antireligieuse du gouvernement de Nankin, qui a moins visé les " minorités " que les temples chinois.

Après l'avènement du régime communiste (1949), les mesures antireligieuses s'attachent à détruire méthodiquement le patrimoine religieux de la Chine, bien que, 
dans le même temps, le pays se dote d'une solide politique de sauvegarde des monuments historiques. En fait, celle-ci ne sera mise réellement en application qu'à partir de 1982 : jusqu'alors, pour un monument remarquable qui est classé, on occulte la destruction de dix autres. Issue comme en Occident d'une lente prise de conscience, la protection du patrimoine en Chine a été plus tardive. En Mongolie-Intérieure, ces mesures sont couplées à la "politique [d'assimilation forcée] des minorités" ${ }^{18}$.

Les années 1950 vont être un peu plus clémentes pour les monastères de Mongolie méridionale que pour les temples chinois : les trois principaux monastères de Kökeqota (le Grand temple, le Petit temple et le Siregetü zuu) restent ouverts jusqu'à la révolution culturelle, alors que tous les temples chinois de la ville sont fermés. La destruction de la culture mongole atteint un point culminant avec le vent de folie qui souffle pendant la révolution culturelle. Des purges d'une ampleur inégalée touchent la moitié de la population mongole adulte, et une part considérable du patrimoine artistique, littéraire et architectural est détruite. Quelques monastères mongols que nous décrirons ci-dessous, fermés à clef et particulièrement protégés, échappent aux pillages, tandis que les autres sont dévastés puis occupés ou rasés. Les statues sont saccagées, les thangkaset les textes religieux sont empilés dans les cours en grands bûchers ${ }^{19}$. Les Gardes rouges tirent à la mitraillette sur les bas-reliefs de bouddhas ornant les murs des stûpas.

24 Après la mort de Mao Zedong (1976), le gouvernement entreprend une " politique en faveur des minorités " - tout en réprimant sévèrement le "crime de séparatisme " et, en 1978, autorise à nouveau les pratiques religieuses. En même temps que les demandes de réouverture de monastères sont étudiées, les principaux édifices religieux sont inscrits au patrimoine historique à la fin des années 1980 et restaurés ${ }^{20}$.

Parmi les quelques bâtiments remarquables de Kökeqota qui ont subsisté, citons le Tabun subury-a ("Cinq stûpas", chin. Wuta), une curieuse pagode sculptée de "mille bouddhas" construite en 1727-1732. Elle prend modèle sur un stûpa de Pékin datant des Ming, lui-même imitation lointaine du temple indien du Mahâbodhi à Bodhgayâ, en Inde, où le bouddha Shâkyamuniconnut l'Éveil. La pagode Tabun subury-a est un grand édifice voûté surmonté de cinq pagodons dont le symbolisme se réfère aux cinq bouddhas transcendantaux. Le monastère qui l'entourait a disparu; la pagode est aujourd'hui un musée bénéficiant d'une protection au niveau national.

Les deux monastères les plus anciens et les plus importants existent toujours et bénéficient d'une protection au niveau régional. Le Grand temple (Jeke zuu), désormais connu sous son nom chinois de monastère Dazhao (Dazhao si) ${ }^{21}$, est périodiquement restauré depuis 1957. Une usine de vêtements s'était installée provisoirement dans ses bâtiments, mais elle a déménagé en 1967 et le monastère est resté fermé à clef pendant la révolution culturelle. L'intérieur de sa salle d'assemblée, qui n'a pas été détruit durant la révolution culturelle, constitue sans doute l'ensemble religieux le plus complet et le plus ancien de Mongolie méridionale. Depuis 1980, le monastère est restauré en moyenne tous les trois ans, et il a subi une restauration particulièrement importante en 1985-1987 (il fut alors entièrement redécoré et repeint). Le Siregetü zuu (chin. Xilitu zhao) a connu à peu près la même histoire (FIG. 3). En 1983, son stûpa, que les Gardes rouges avaient tenté de démolir sans succès, a été fouillé ; on y a découvert des vêtements, des sûtras datant des Ming et des objets divers. Le monastère a été somptueusement (sur)restauré dans les années 1980. Toutes ces restaurations sont effectuées par des charpentiers han spécialisés dans la construction traditionnelle 
chinoise. Ils n'ont aucun mal à restaurer les édifices religieux de Kökeqota, quoique ceux-ci comportent des éléments décoratifs tibétains, mais employent toujours des techniques et des matériaux chinois. Cependant, outre l'aspect clinquant des décorations récentes, on remarque des rajouts d'élémentsnouveaux, et surtout d'éléments chinois qui ne correspondent pas toujours à des restaurations, comme la balustrade de marbre entourant le stûpa du Siregetü zuu.

FIG. 3 - Tournage d'un film sur les danses rituelles cam au Siregetü zuu

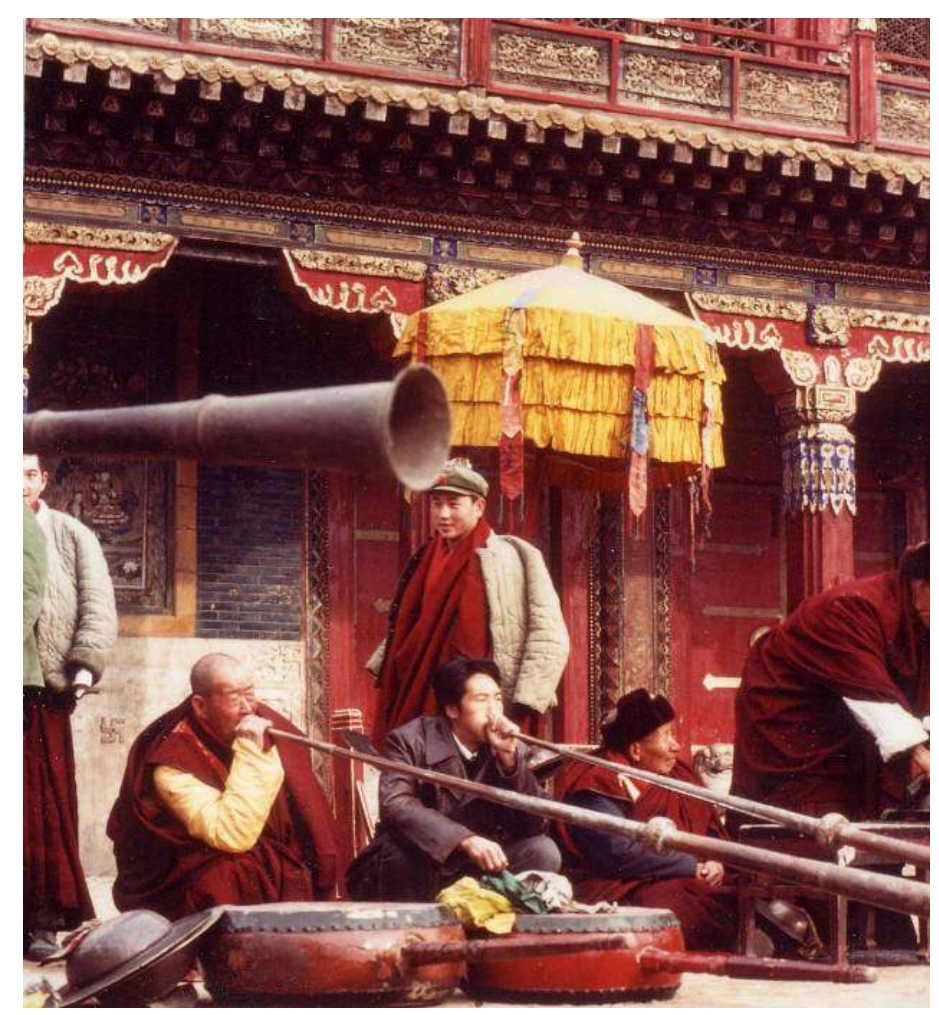

Les acteurs sont des soldats de l'armée rouge

Roberte Hamayon, 1980

La pratique de reconvertir un édifice ancien en usine, école ou entrepôt a paradoxalement permis de sauver des bâtiments, dont certains sont aujourd'hui rendus au culte, tel le Grand temple. En revanche, certains édifices reconvertis ont été progressivement modifiés pour répondre à leur nouvelle fonction ; au bout de quelques décennies, ces anciens bâtiments ne sont plus jugés fonctionnels et sont rasés. C'est ce qui est arrivé au troisième plus grand monastère de Kökeqota, le Petit temple (Baya zuu, chin. Xiaozhao, fondé au début du XVII siècle) : restauré en 1949, il fut en grande partie détruit pendant la révolution culturelle ; quant aux bâtiments restants, occupés par une école, ils ont été peu à peu abattus, leur entretien coûtant trop cher, et il ne reste plus aujourd'hui du Petit temple que son beau portique triomphal. Similaire a été le destin du Corzi-jin zuu, transformé en école. Ce monastère fut inscrit parmi les bâtiments protégés au niveau régional car il avait abrité des activités du Guomindang en 1925, mais cette protection a été insuffisante : il n'en reste plus que le pavillon d'entrée et une salle arrière. La salle d'assemblée principale du Emci-jin zuu (Naimuqi zhao) a subsisté, et est actuellement occupée par une usine de verre: elle subira probablement le même sort si les autorités ne délogent pas l'usine. Les autres 
monastères intra-muros n'existent plus, en dehors de quelques petits bâtiments réaffectés. Les cinquante-six temples chinois recensés en 1949 dans l'ensemble de la capitale ont été détruits ou abandonnés pendant la révolution culturelle; seul aurait été préservé le temple de la littérature des Tümed, dont les bâtiments sont actuellement inclus dans l'École des Tümed (Tumote xuexiao).

\section{La reprise des activités religieuses}

Les deux grands monastères de Kökeqota ont été rendus au culte ${ }^{22}$. Le Onzième Siregetü qutuүtu (né en 1943) ${ }^{23}$, aujourd'hui la principale réincarnation de MongolieIntérieure et le personnage central de la renaissance du bouddhisme dans la Région autonome, s'est dépensé sans compter pour la réouverture des monastères intra-muros et extra-muros de Kökeqota. Intronisé en 1963, il a été réhabilité après la révolution culturelle. Il dirige l'Association bouddhique de Kökeqota ${ }^{24}$, reconstituée depuis 1980 et logée dans l'ancienne résidence de sa lignée au Siregetü zuu. L'Association contrôle les activités religieuses et s'assure que la politique officielle est respectée. Dans les années 1980, le Siregetü qutuүtu a entrepris la restauration et dirigé les rituels de consécration du Grand temple, du monastère Üsütü (chin. Wusutu zhao) à $12 \mathrm{~km}$ à l'ouest de Kökeqota, du monastère Sir-a mören (chin. Puhui si) dans la bannière des Darqan et Mayu mingyan et du monastère du Beile (chin. Bailing miao) dans la bannière des Dörben keüked (chin. Siziwang). Les moines qui s'y réinstallent reçoivent des indemnités, et les biens confisqués sont restitués à condition que les communautés puissent prouver la perte subie ${ }^{25}$. L'une des conditions de réouverture d'un monastère est qu'il soit économiquement autonome, c'est-à-dire capable d'attirer les donateurs vivant à proximité et les touristes. Il est interdit de détruire les structures existantes, et la responsabilité de l'entretien et de la réparation des bâtiments échoit à la communauté monastique. Celle-ci se doit de suivre les directives des administrations en charge du patrimoine (bureaux du Patrimoine, de l'Urbanisme, de l'Archéologie et autres administrations concernées), et toute rénovation, reconstruction ou extension doit être approuvée au préalable. Les communautés dépendent bien de la hiérarchie religieuse, mais les bâtiments, avant d'être des édifices de culte, sont considérés comme “patrimoine culturel”, propriété de l'État, sous la juridiction des différents bureaux administratifs chargés de la protection du patrimoine. Or ceux-ci, soutenus par des autorités locales soucieuses de développer le tourisme, entrent parfois en conflit avec les autorités religieuses. Ainsi, l'Association bouddhique de Kökeqota avait décidé en 1987 de repeindre la salle d'assemblée du Grand temple sans en avertir les autorités. Les conservateurs du département archéologique du musée de Mongolie-Intérieure, avertis trop tard, ne réussirent à sauver qu'une partie de l'ancien décor : les peintures furent découpées dans l'urgence avec leur support et ont été entreposées dans le musée d'Histoire de la ville (Gongzhu fu, voir ci-dessous). Les peintures nouvelles, de mauvaise qualité, sont déjà très abîmées en 2002.

Prises en charge par l'État, par la RAMI et par les collectivités locales jusqu'au milieu des années 1990 (l'État a dépensé 15 millions de yuans entre 1985 et 1995 pour le patrimoine de Mongolie-Intérieure), les réparations et restaurations sont aujourd'hui principalement entreprises par les communautés religieuses, sous la direction de l'administration locale ou régionale qui peut financer une partie des travaux. Le financement est complété par des collectes auprès de la population, et pas seulement 
auprès de la population mongole. Les monastères de Kökeqota sont aujourd'hui patronnés par les Mongols et par les Han. Ces derniers manquent en effet de temples, car la ville ne comprend aujourd'hui que deux petits lieux de culte chinois : le Guanyin miao, petit temple bouddhique dédié à Guanyin, dans la Vieille ville, reconstruit en 1987-1988 par les autorités de Kökeqota au huitième de sa taille d'origine et rendu au culte (son entrée fait face au nord, ce qui est tout à fait inhabituel), et un petit monastère taoïste abritant quelques moines et nonnes, reconstruit dans la Nouvelle ville.

Depuis ma première visite en 1993, j'ai pu constater les changements et revers de fortune des deux monastères mongols. Le Grand temple n'était alors que l'ombre de son rival et ressemblait bien plus à un musée gardé par trois moines égarés qu'à un monastère. Au contraire, le Siregetü zuu débordait de ferveur religieuse. Les Han le fréquentaient particulièrement (en 1995-1998, l'association bouddhique de laïcs Jushilin louait dans ce monastère une chapelle dédiée à Guanyin après l'apparition de " miracles" : deux statues émirent de la lumière et un papillon estival apparut en hiver. Outre les lamas, les festivals de ce monastère rassemblaient moines bouddhistes han, laïcs du Jushilin et religieux taoïstes, qui " concélébraient" dans la tradition impériale des "Trois religions se rejoignant" ${ }^{26}$ ). En 1995, le Siregetü zuu comptait une trentaine de moines, les plus jeunes ayant une dizaine d'années, et célébrait deux festivals religieux par an, dont un rituel de danse cam au Nouvel an. En 1999, on comptait 20 moines; les plus jeunes avaient 12-13 ans; le monastère organisait près de dix festivals par an. La réputation des moines était cependant assez mauvaise. Ils roulaient en moto dans la ville, possédaient des téléphones portables; on les accusait même de fréquenter des femmes dès la nuit tombée. En 1995, un moine tibétain de l'Amdo y était même " exilé ", en punition pour avoir trop voyagé.

31 En 2002, le Siregetü zuu n'a plus la faveur des fidèles et ne rassemble plus que dix moines : les autres sont, paraît-il, rentrés chez eux. Le monastère n'est presque plus fréquenté et n'organise plus de fête; la chapelle de Guanyin a été transformée en boutique et seuls quelques touristes visitent encore ce site. Un vieux moine hargneux les dissuade de s'arrêter dans la salle d'assemblée, de regarder les statues de près et d'y rentrer une deuxième fois.

Au contraire, le Grand temple connaît une nouvelle vie, et toute l'activité religieuse de Kökeqota semble y avoir déménagé. En 1995, il organisait deux festivals par an, dont un rituel cam au Nouvel an. En 1997-1999, on a reconstruit une arche commémorative devant le pavillon d'entrée, ainsi que les tours de la Cloche et du Tambour dans la première cour. La communauté monastique a augmenté avec sa fortune nouvelle (30 moines en 1999). La restauration est marquée par une stèle commémorative en chinois (sous le portique triomphal), datée de 1997, comportant les noms des nombreux donateurs gravés sur sa face sud : associations, sociétés et particuliers. Le Grand temple a organisé en 2002 une dizaine de fêtes religieuses, dont le grand cam du Nouvel an, le festival de Maitreya (le 15 du $\mathrm{I}^{\mathrm{er}}$ mois lunaire), la récitation du Kanjur (du 8 au 15 du

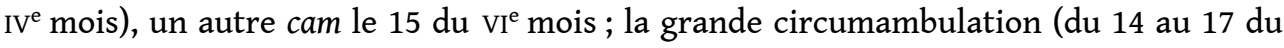
$\mathrm{VIII}^{\mathrm{e}}$ mois), l'anniversaire de Tsongkhapa (le $25 \mathrm{du} \mathrm{x}^{\mathrm{e}}$ mois), un rituel aux obuy-a ${ }^{27} \mathrm{des}$ alentours de Kökeqota le $13 \mathrm{du} \mathrm{v}^{\mathrm{e}}$ mois, etc. La communauté reçoit sans cesse des personnalités religieuses et politiques, de Chine et de l'étranger; la foule se bouscule pour brûler de l'encens. De très jeunes moines jouent dans la cour; tous portent baskets et jean sous leur robe monastique. Les drapeaux et les moulins à prières, 
interdits pendant longtemps, ont refait leur apparition (FIG. 4). Dans la grande salle d'assemblée, les moines écrivent sur un tableau les noms des donateurs de passage, Chinois du continent, Mongols, Taïwanais, etc. (les sommes moyennes sont de l'ordre de 5 à 10 yuans, mais peuvent aller jusqu'à 200 yuans). Le temple occidental du monastère, dédié aux divinités protectrices, est en cours de restauration " dans son état initial " : on a reconstruit une grande salle à étage au nord, large de sept travées, et des halls latéraux. Le toit de cette grande salle est décoré de deux dragons jouant avec une perle, ornement jamais vu précédemment sur les temples mongols.

FIG. 4 - Salle d'assemblée du Grand temple (Jeke zuu)

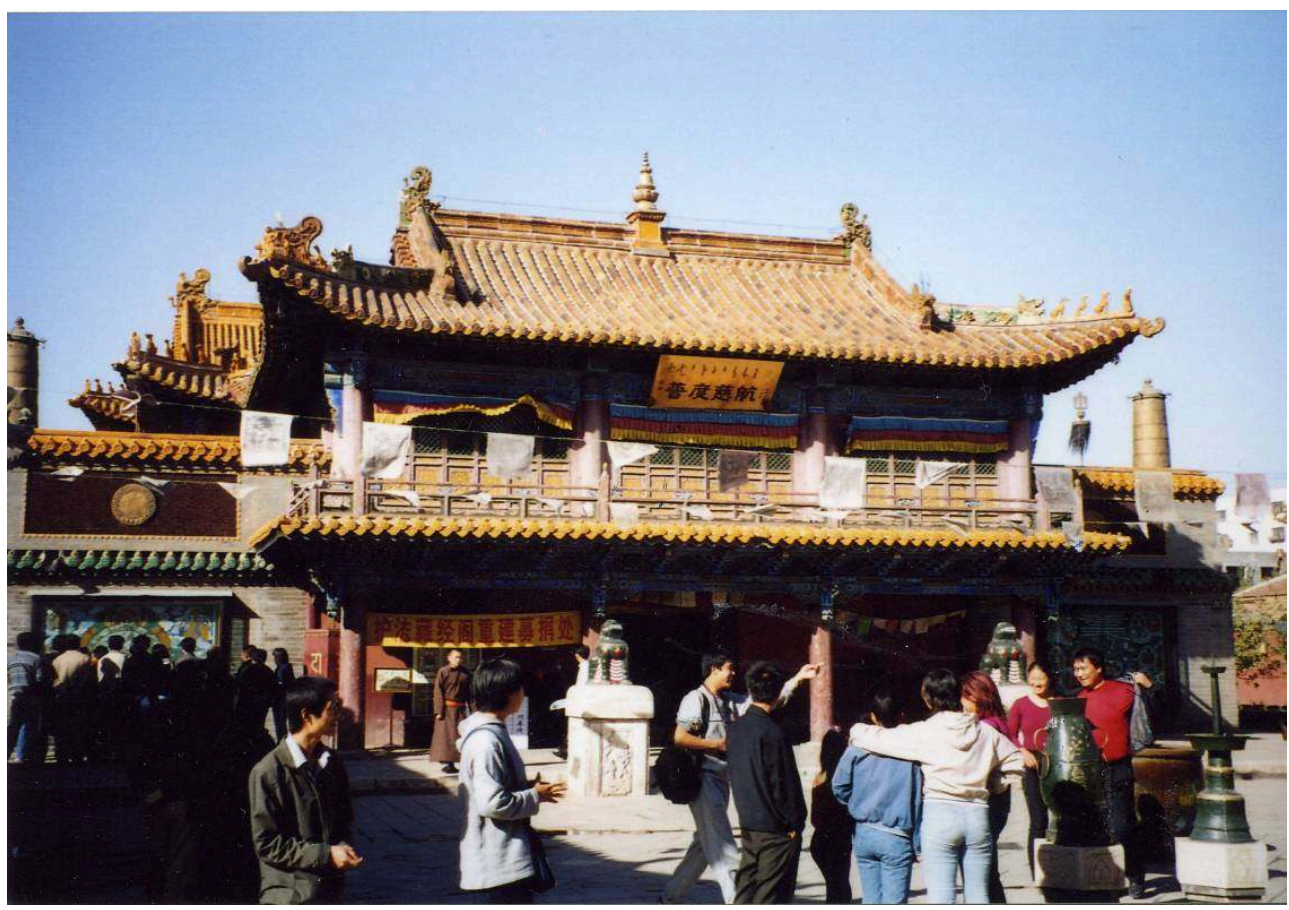

Sous le porche, une banderole jaune écrite en chinois incite à donner des offrandes pour réparer - en réalité reconstruire - le pavillon de la bibliothèque de l'aile occidentale

Isabelle Charleux, 2002

Les deux monastères sont envahis par une dizaine de boutiques. Les fidèles y achètent, pour offrir aux monastères, des statuettes de style chinois représentant des bodhisattvas (Guanyin chinoise) et surtout des divinités populaires chinoises, comme la divinité martiale Guan Yu ou le dieu des richesses (Caishen). On trouve même un petit autel à Caishen et des Guanyin en porcelaine à l'intérieur du vieux temple occidental du Siregetü zuu. Les autels des monastères mongols sont ainsi colonisés par ces dieux chinois (FIG. 5). 
FIG. 5 - Statue de Tsongkhapaet de ses disciples à l'intérieur d'une chapelle du Jeke zuu

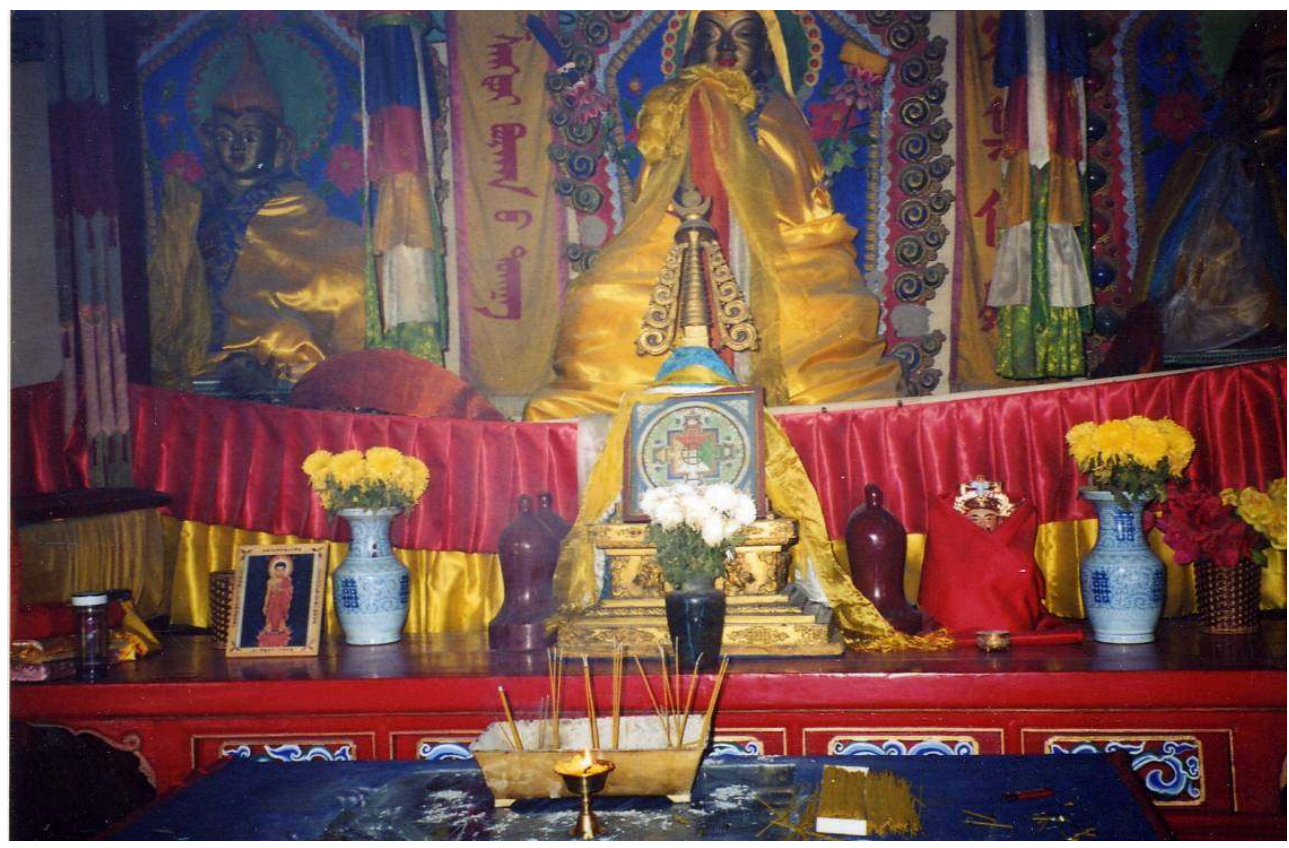

Sur la table d'offrandes, on remarque, à droite, la statuette d'une divinité chinoise, couverte d'un tissu rouge, et, à gauche, une reproduction moderne chinoise du bouddha Shâkyamuni. Toutes les inscriptions sont bilingues (mongol et chinois)

Isabelle Charleux, 2002

Les temples et monastères extra-muros sont également ouverts au tourisme depuis peu. Le Caqar blam-a zuu, temple principal du Üsütü zuu à $12 \mathrm{~km}$ à l'ouest de la ville, qui abrite depuis 1991 la seule école monastique de Mongolie-Intérieure, a rouvert aux touristes en 2001. Le monastère de la Grotte des lamas (Blam-a-jin aүui, chin. Lamadong), ancien ermitage de montagne à $30 \mathrm{~km}$ à l'ouest de Kökeqota, a été restauré. Une salle d'assemblée a été reconstruite en 1998-1999 : c'est aujourd'hui une charmante excursion pour les citadins. La population de la ville se déplace une fois par an pour assister à des sacrifices aux obuy-a, où officient des lamas : à l'obuy- $a$ Wugongba (Wugongba aobao) dans les monts Daqing, près de la ville, et à l'obuy-a Huzhaicun (Huzhai cun aobao), dans la banlieue ouest (chaque année le $13 \mathrm{du} \mathrm{v}^{\mathrm{e}}$ mois).

La communauté Hui a réinvesti la principale mosquée à l'élégant minaret de $33 \mathrm{~m}$ de haut bâtie au nord de la Vieille ville en 1789 et classée au patrimoine régional, qui s'est récemment agrandie d'un grand bâtiment moderne (FIG. 6). J'ai vu en 2002 une autre petite mosquée logée dans les bâtiments anciens à proximité du Emci-jin zuu. Une église catholique est également en activité au nord de la Vieille ville. 


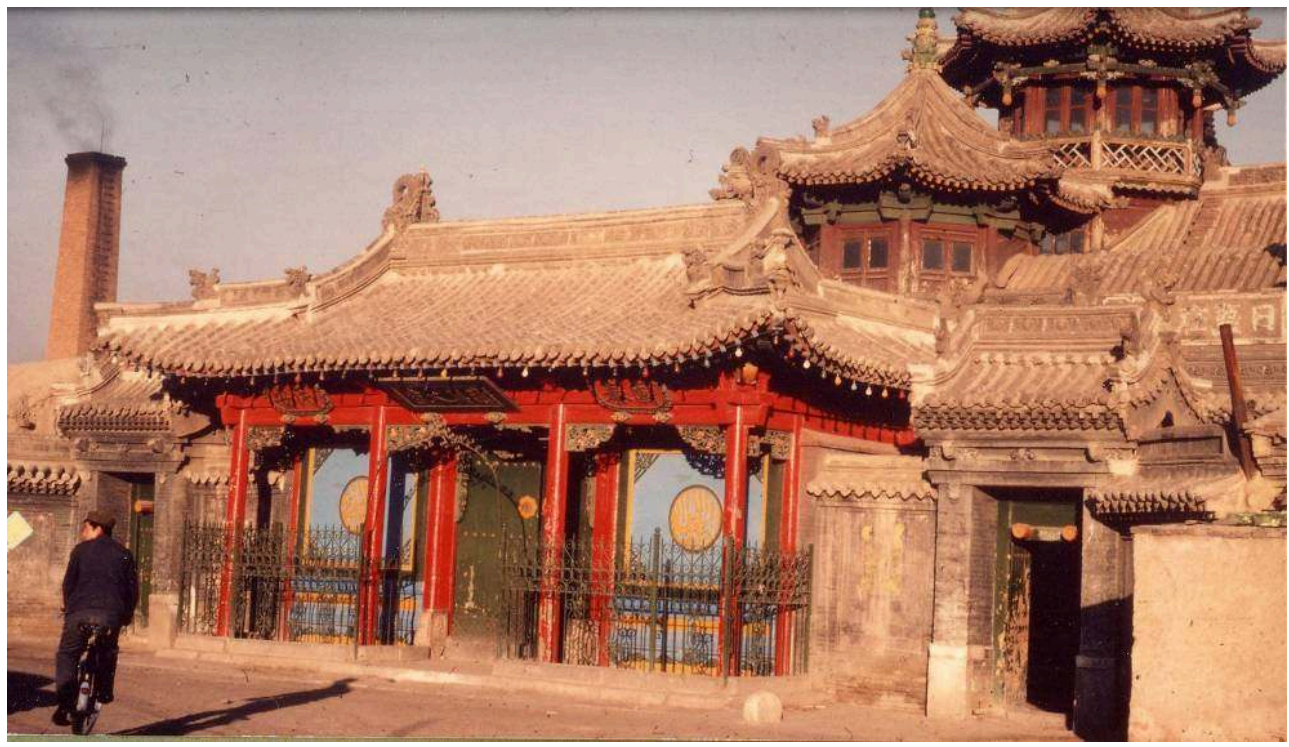

Roberte Hamayon, 1980

\section{Protection et début de restauration de la Vieille ville}

Après avoir d'abord concerné les monuments les plus anciens et les plus prestigieux - comme cela a aussi été le cas en France -, la protection du patrimoine s'est étendue au patrimoine urbanistique, puis au patrimoine "implicite vécu" (musique, arts, artisanat, traditions festives locales). La protection des "villes célèbres historiques et culturelles " a été mise en œuvre à partir de 1982, et celle des "secteurs historiques " à partir de $1991^{28}$. Malheureusement, il est souvent déjà trop tard pour préserver des quartiers anciens dans la plupart des villes de Chine: l'habitat traditionnel et les murailles, jadis condamnés en tant que symboles des aspects indésirables de la culture traditionnelle, continuent à être des cibles de destruction plutôt que de préservation.

Or, la capitale de la Mongolie-Intérieure conservait encore dans les années 1990 un des ensembles urbains les plus intéressants de la Chine du Nord. Elle fut classée en 1987 sur la liste des "villes célèbres historiques et culturelles " protégées à des fins touristiques au niveau national. Les restaurations ont alors porté sur les édifices de trois rues partant du Grand temple: vers le sud, la rue Dazhao qianjie (" rue avant du Grand temple ") ; vers l'est, la rue qui serpente en direction de l'axe Dananjie ; vers l'ouest, la rue Tongshun. Ces rues formaient depuis la fin du $\mathrm{xvI}^{\mathrm{e}}$ siècle le vieux quartier de commerce et d'amusement (théâtre, cirque, maisons de thé, restaurants, marché aux puces, etc.), actif jour et nuit. Juste au sud du portique triomphal du monastère, une "source de jade", composée de neuf puits qui, selon la légende, avaient jailli sous le sabot du cheval de l'empereur Kangxi, était réputée pour son eau miraculeuse censée guérir cent maux (on la donnait à boire aux parturientes et aux personnes ivres) ${ }^{29}$.

La campagne de restauration de ces trois rues a débuté en 1984 (avant même le classement), et s'est poursuivie jusqu'en 1988. En 1987, le gouvernement de la ville, grâce à des fonds spéciaux du gouvernement national, a entrepris la restauration des vieilles boutiques avec des techniques traditionnelles, pour leur rendre leur apparence des années 1920. Des travaux ont aussi été effectués dans d'autres rues comme 
Tongxuntan. Cet ensemble urbain a été comparé, pour sa valeur, aux vieux quartiers de Shanghai et de Nankin. Lors de ma première visite en 1993, je trouvai une vieille ville très bien conservée. La célèbre " rue avant du Grand temple " (350 m de long sur 6 à $7 \mathrm{~m}$ de large) faisait le bonheur des touristes qui se croyaient réellement au début du $\mathrm{xx}^{\mathrm{e}}$ siècle, avec ses échoppes chinoises à étage servant de pharmacies, de boucheries et de boutiques vendant toutes sortes d'objets de la vie quotidienne; toutes ces boutiques avaient conservé leur ancien nom. Les boutiques de la rue partant vers l'est vendaient de l'encens et des objets de culte. La rue Tongshun regorgeait de délicieuses gargotes proposant du gutou (porc bouilli sur os), des shaomai (spécialité de raviolis), des lamian (nouilles étirées), des shaodaomian (nouilles coupées au couteau) et des filets de poisson. Des paysans venaient vendre les produits de la campagne. La plupart des bâtiments dataient des Qing, quelques-uns des Ming, d'autres de la République. Le bâtiment typique était à deux niveaux, et trois fois plus profond que large, avec le magasin sur la rue, et les quartiers d'habitation derrière. Les toitures étaient à deux pentes couvertes de tuiles grises ou vernissées avec une arête faîtière décorée. Les échoppes les plus riches offraient de magnifiques façades en bois travaillé, avec des fenêtres à lattis de bois, de riches peintures, des décorations en céramique et pierre sculptée. Chaque boutique présentait un style un peu différent.

Dans le reste de la Vieille ville, le dédale de ruelles étroites, plus résidentielles et bordées de vieilles maisons de briques ou de torchis, les beaux porches voûtés ouvrant sur des cours où pénétraient jadis les caravanes de chameaux, et la vie intense du quartier donnaient à l'ensemble un charme désuet mais plein de vie (FIG. 7 et 8). Dans la Vieille ville et dans le quartier Hui, on trouvait plutôt des constructions dans le style du Shanxi : de petites maisons de briques ou de pisé avec toit en terrasse ou faiblement pentu sur l'avant. De nombreux édifices isolés étaient autrefois des maisons sur cour (la cour et les bâtiments sur rue ayant été supprimés lors de l'élargissement des rues, les bâtiments du fond, qui avaient seuls subsisté, se retrouvaient en bordure de la nouvelle rue) ${ }^{30}$. La plupart des rues étaient en terre et se transformaient en bourbier aux premières pluies. 
FIG. 7 - Vue de la Vieille ville de Kökeqota depuis la pagode Tabun subury-a

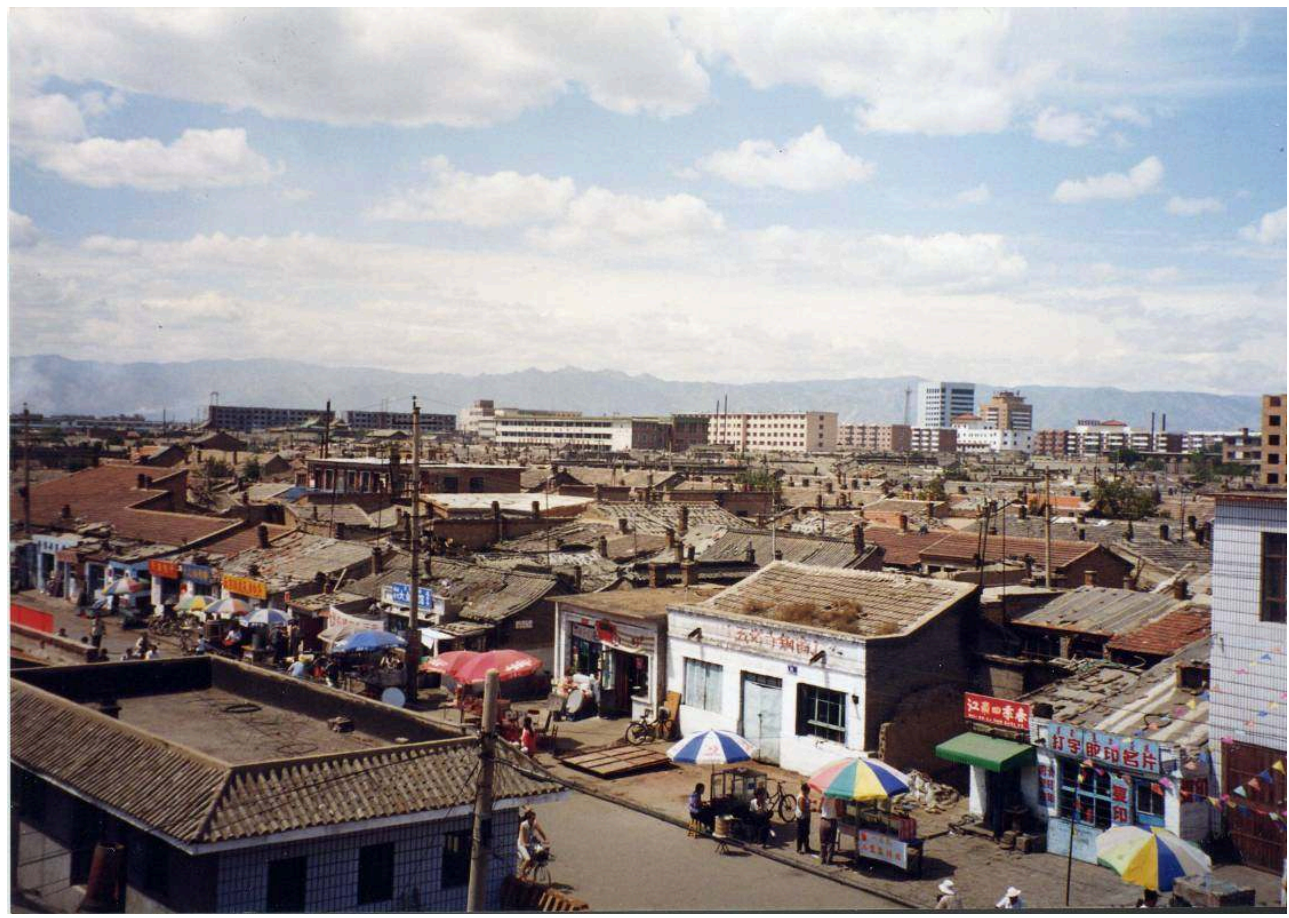

Le tissu urbain est serré ; au nord, les tours marquent la limite de la Vieille ville Isabelle Charleux, 1999

FIG. 8 - Boutique formant angle, rue avant du Grand temple (Dazhao qianjie)

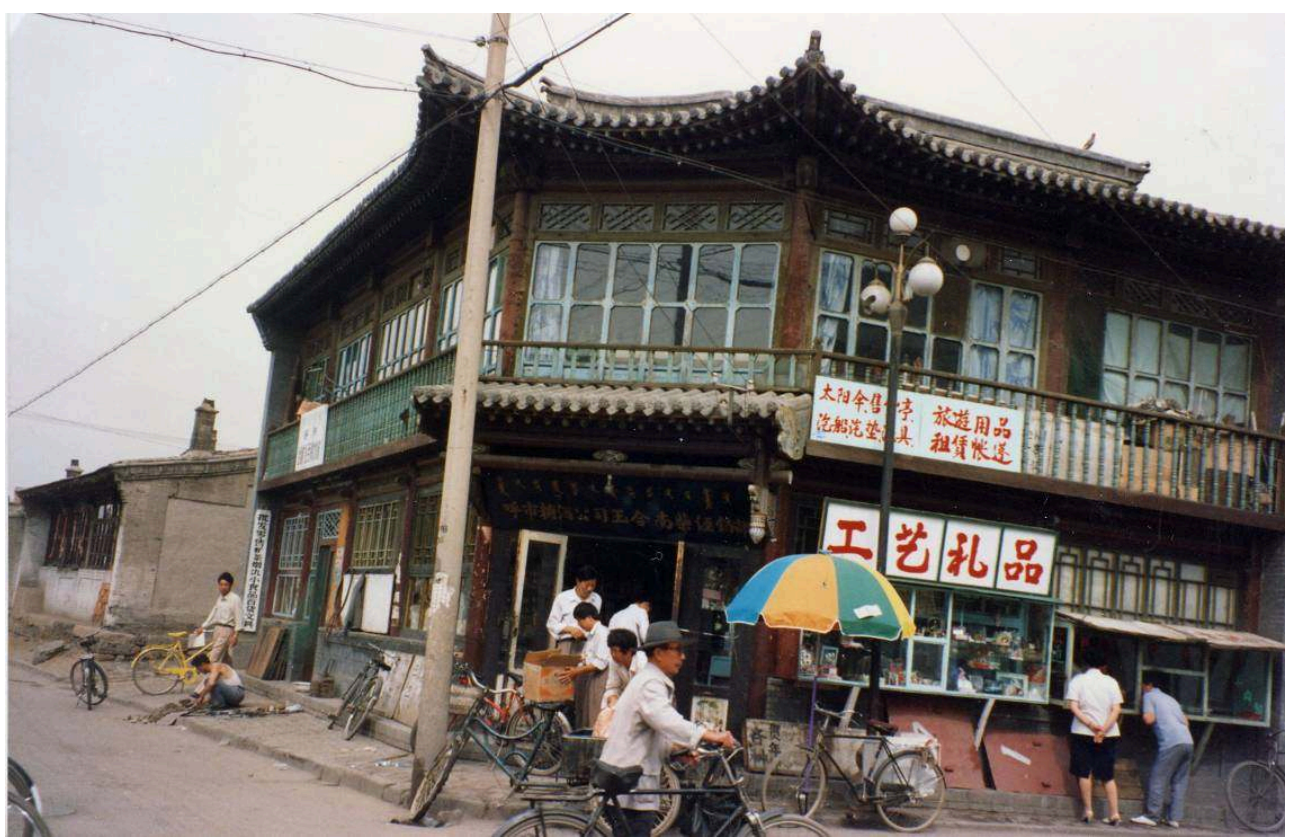

Isabelle Charleux, 1998

Kökeqota n'était pas avant la fin des années 1990 une destination touristique importante, et les nouveaux quartiers continuaient de se construire entre les deux villes anciennes. Les destructions des années 1950 avaient essentiellement touché la 
Grande rue sud (Dananjie) que l'on avait alors élargie ; la plupart des bâtiments qui la bordent sont de cette époque.

\section{7-2002: La destruction de la Vieille ville}

41 En 1997 débute la destruction de deux des rues partant du Grand temple, vers le sud et vers l'est respectivement, pourtant classées “patrimoine historique" 31. En 2002, elles sont entièrement rasées, après que les habitants ont été expulsés sans ménagement (FIG. 9 et 10).

FIG. 9 - Façade sud du Jeke zuu, montrant une partie de la Vieille ville qui vient d'être rasée

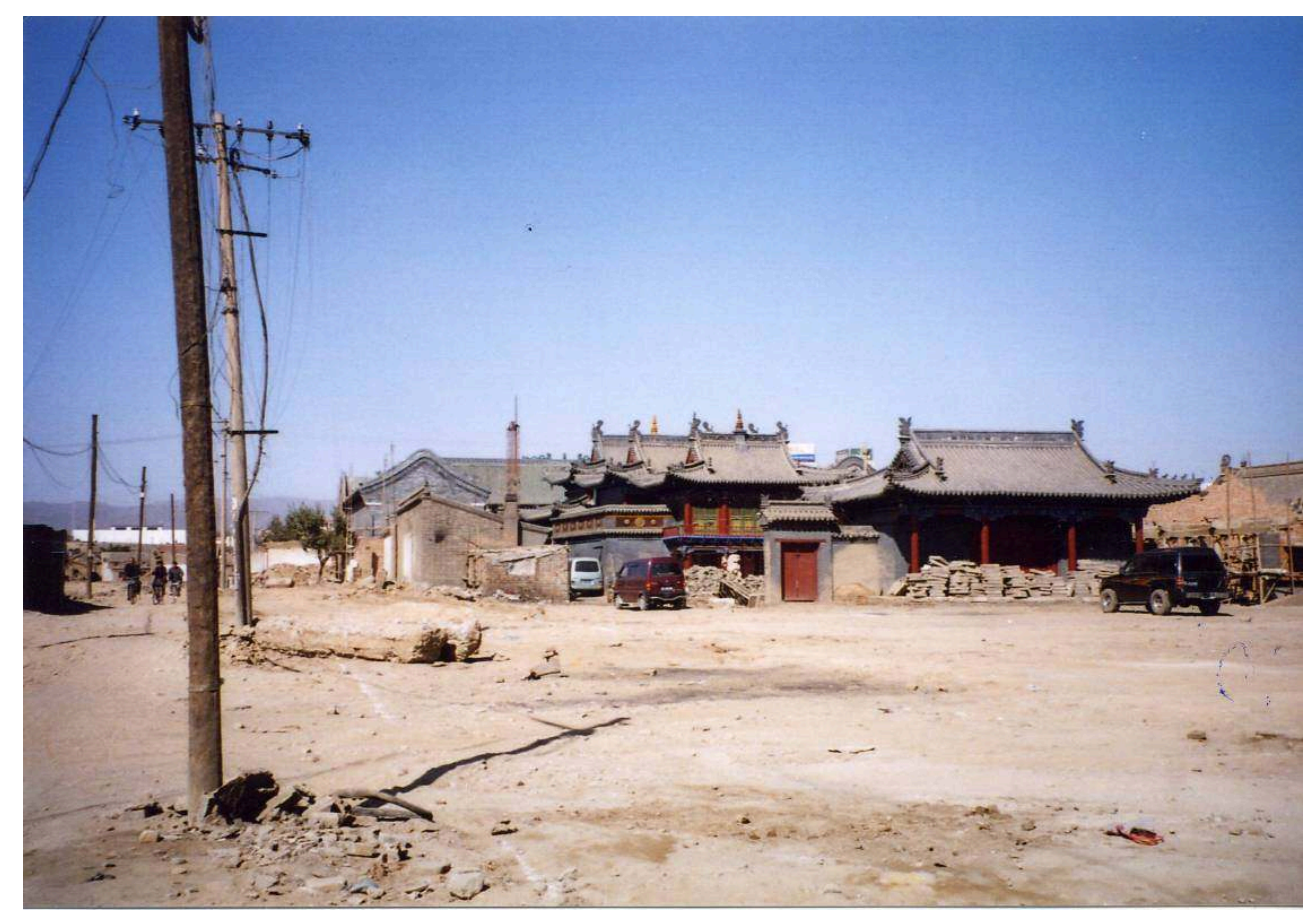

Isabelle Charleux, 2002 
FIG. 10 - Abords du Jeke zuu

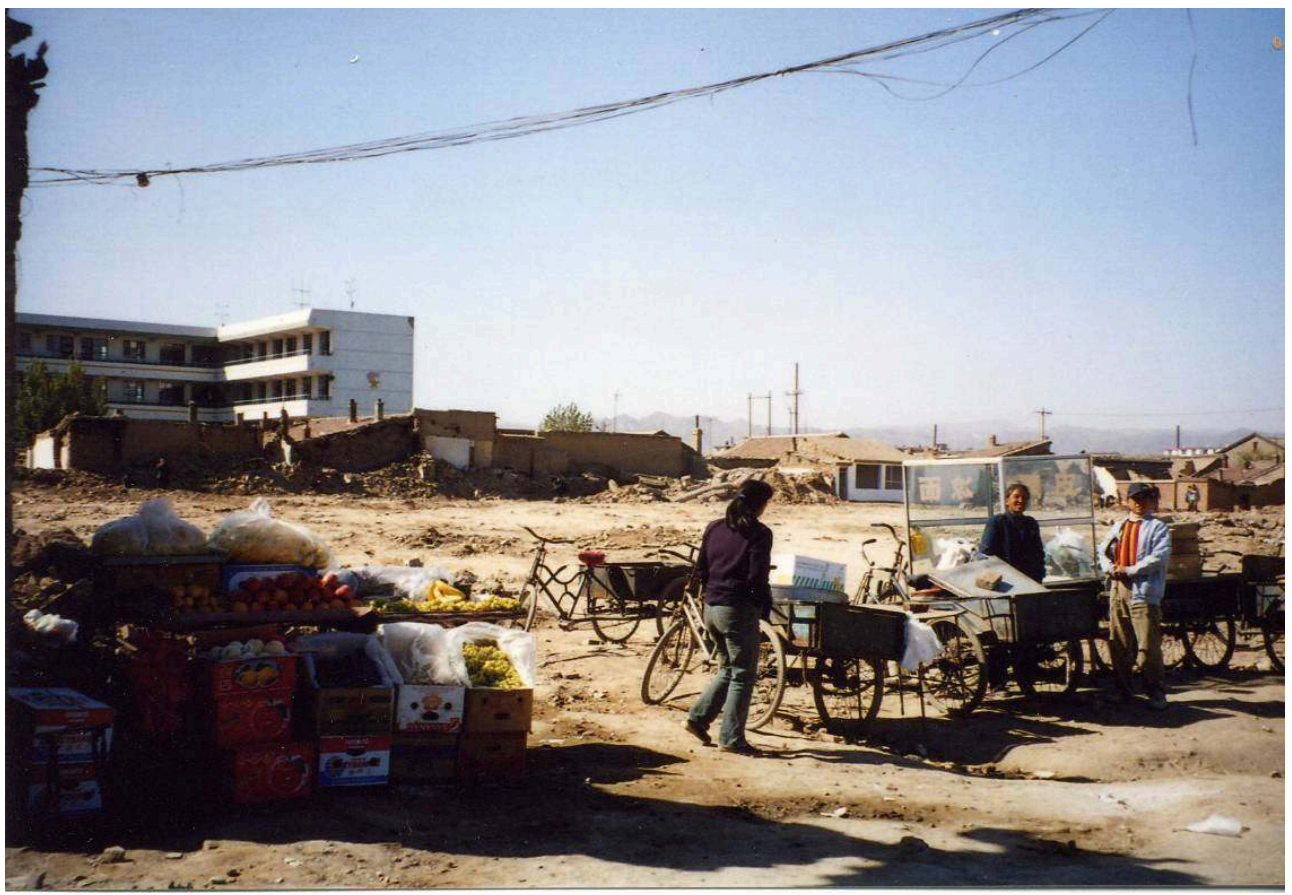

Isabelle Charleux, 2002

On y reconstruit des bâtiments standardisés, à trois niveaux, en briques et en béton, tous identiques (infiniment moins beaux que les pastiches d'architecture ancienne que l'on peut voir à Pékin ou à Tianjin ${ }^{32}$ ), dédiés à des commerces touristiques (FIG. 11).

FIG. 11 - Nouvelle rue avant du Grand temple aux bâtiments standardisés, triste pastiche de l'architecture ancienne

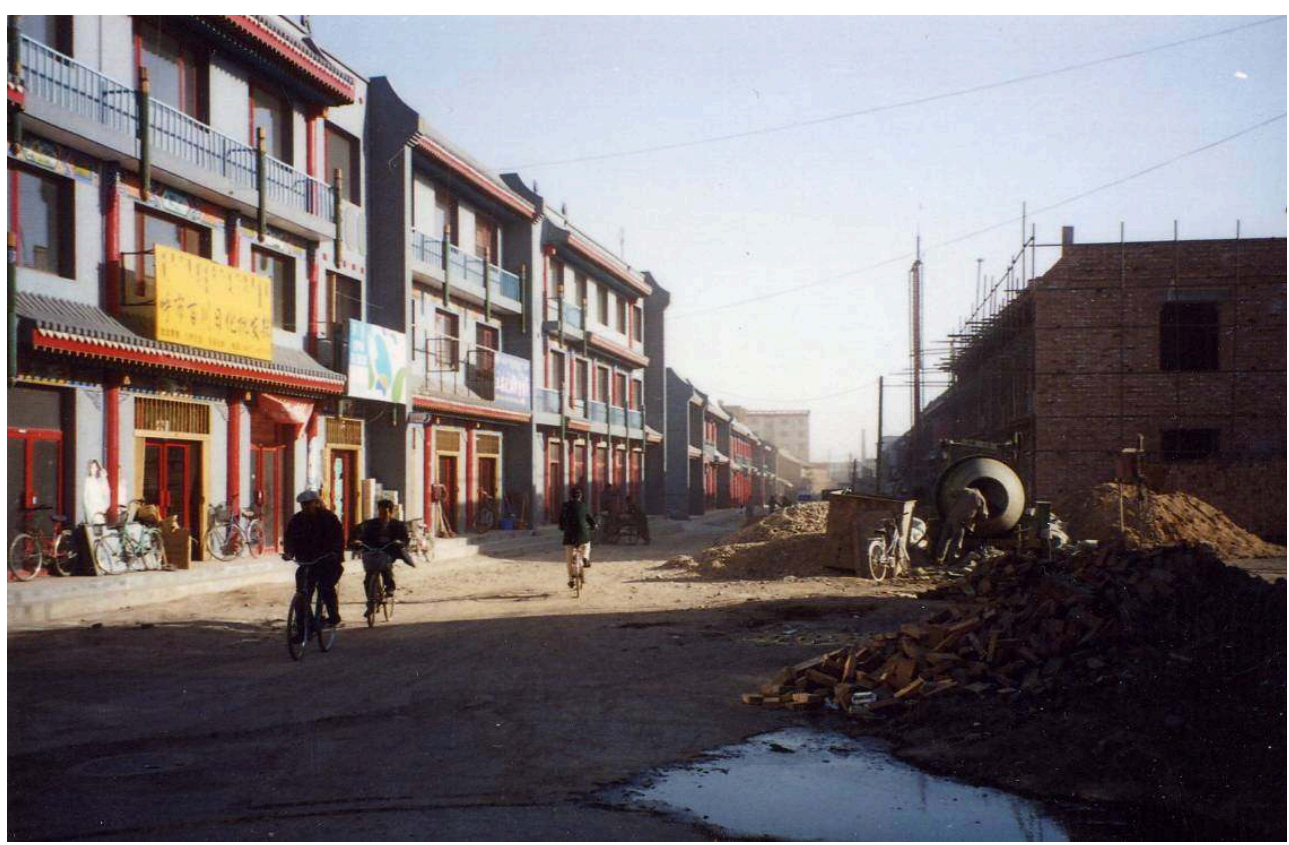

Isabelle Charleux, 2002 
Seule une petite portion de la rue Tongshun a été restaurée à des fins touristiques. Un gros restaurant chinois remplace les dizaines de gargotes qui entouraient le temple. Le reste de la Vieille ville n'a pas échappé aux destructions : en septembre 2002, les deux tiers de la Vieille ville étaient en train d'être rasés par les bulldozers et les rues n'étaient qu'un champ de briques. Le moindre souffle de vent se change en tornade de poussière. Les quelques îlots épargnés n'en ont plus pour longtemps. Seuls les deux grands monastères émergent tristement de ce désert de ruines que les ouvriers déblaient pierre par pierre, brique par brique. Bientôt les monastères seront cachés par de hauts buildings, et les larges avenues auront tout oublié des anciennes ruelles. Au sud du Siregetü zuu, on perce une grosse artère bordée de bâtiments à deux étages, sans âme, tous identiques. Une place est dégagée devant le Grand temple, sous l'arche commémorative, pour servir de parking. La "source de jade" avait en 1999 été entourée d'une petite enceinte dédiée à un roi-serpent. Bien que son eau soit polluée depuis longtemps, on venait y boire du thé en plein air (2 yuans l'entrée, plus le thé). En 2002 , l'ensemble a été rasé à l'exception des neuf puits.

\section{La croissance démographique et économique}

En 2002, la ville explose. Elle couvre désormais plus de $86 \mathrm{~km}^{2}$ pour une population de 710000 habitants $\left(17224 \mathrm{~km}^{2}\right.$ pour une population de 2 millions 44 mille habitants dans toute la municipalité de Kökeqota). Les tours de verre bleuté s'élèvent à une vitesse vertigineuse. Des immeubles modernes prestigieux (bâtiments administratifs, hôtels) adoptent des formes originales. Certains sont couronnés de dômes-yourtes - tel le bâtiment du comité permanent du Congrès populaire national de la RAMI, bloc rectangulaire de cinq étages couronné d'un dôme de tuiles blanches à motifs bruns. D'autres sont, comme dans le reste de la Chine, surmontés de pavillons chinois ou de constructions de formes diverses (boule, petite arche commémorative), tels la tour de la Banque de Chine et l'immeuble de la Bibliothèque de Mongolie-Intérieure. Curieusement, c'est le modèle soviétique d'une tour en flèche entourée de quatre tours plus petites qui a été choisi pour un des grands bâtiments du nouveau "quartier en développement " de Jinchuan, datant de la fin des années 1990.

La Chine a de grands projets pour la Mongolie-Intérieure, qui est incluse dans le “programme de développement des régions de l'Ouest" [de la Chine]. On note un véritable souci de l'environnement, puisque sa dégradation menace toute la Chine du Nord : sept projets sont en cours, comprenant des reforestations, la lutte contre les inondations, et... l'abandon de pâturages (pour préserver l'environnement!) ${ }^{33}$. Pour Kökeqota, les projets concernent le développement des infrastructures routières (une nouvelle autoroute Kökeqota-Baotou, doublant la route existante, a été ouverte en 2000, et deux ponts routiers ont été édifiés en ville pour réduire les embouteillages), le développement de l'approvisionnement en électricité et en eau, le traitement des ordures et des eaux usées, le contrôle de la pollution atmosphérique (la ville est particulièrement polluée de décembre à février, lorsque les bâtiments sont chauffés, le charbon étant la principale source d'énergie) et la création de parcs et d'habitations "économiques". Un des objectifs est l'augmentation du niveau de vie qui devra atteindre un niveau " confortable" en 2005 et rejoindre la moyenne du pays en 2015. Un programme de détournement de l'eau du fleuve Jaune afin de satisfaire les besoins futurs de la ville a commencé en août 1998 grâce à un prêt japonais ${ }^{34}$. 
Certes, les vieux logements n'offrent aucun confort et les avenues connaissent des embouteillages. De plus, il est trois à cinq fois plus cher de restaurer ou construire à l'ancienne que de bâtir du neuf, et la seule justification aux yeux des autorités en est le tourisme culturel. Par ailleurs, les démolitions sont souvent exécutées trop rapidement pour qu'une intervention quelconque puisse les empêcher. C'est pourquoi la Vieille ville de Kökeqota est sévèrement menacée par les promoteurs, qui au plus grand mépris du contexte architectural ont rasé les “zones protégées" enserrant les monuments historiques. On ne s'est même pas donné la peine de faire des relevés, des plans détaillés et des recensements des édifices anciens (en dehors de ceux qui sont ou étaient protégés), et il existe très peu d'archives photographiques.

Les habitants n'ont pas vraiment le choix. Même lorsqu'on n'emploie pas de moyens musclés pour les expulser, les prix de l'immobilier augmentent; les menaces d'expropriation ont pour résultat que les habitants ne font plus de travaux et que les maisons tombent d'elles-mêmes en ruine. Les gens interrogés sur cette situation répondent : ces maisons sont vieilles et insalubres, qui voudrait encore y habiter ? elles n'ont pas de sanitaires, pas de salle de bain, et le coût de leur restauration et de leur modernisation dépasse largement le profit réalisé quand on construit du neuf.

\section{L'explosion du tourisme}

ville est partagée entre sa croissance démographique et économique, qui entraîne une forte poussée immobilière, et sa nouvelle vocation touristique. Les touristes ne sont plus seulement les Chinois d'Outre-mer. Les Chinois du continent ont vu leurs vacances rallongées depuis quelques années, passant pour une grande partie d'entre eux d'une à cinq semaines par an ; leur portefeuille étant mieux garni, ils consacrent leurs semaines de loisir à visiter les hauts lieux touristiques du pays. Kökeqota, à une nuit de train seulement de la capitale, est une destination de choix pour les Pékinois.

La ville fait effectivement tout pour attirer les touristes. Des guides touristiques par province et par ville - genre littéraire nouveau en Chine - trônent sur les éventaires des librairies ${ }^{35}$ et le nombre d'hôtels a explosé. Les musées se multiplient depuis 2001-2002. Citons le yamen du général (en fait, du gouverneur) de Suiyuan (Jiangjun yashu) comme exemple de préservation à travers une reconversion. Ce complexe construit entre 1737 et 1739 est situé juste à l'ouest du centre de la Ville nouvelle. Il servit de résidence au gouverneur jusqu'en 1921, de quartiers généraux du gouvernement régional jusqu'en 1954 (sous les nationalistes puis les communistes) et de Cour suprême de la région autonome de Mongolie-Intérieure de 1956 jusqu'au milieu des années 1980. Bien conservé, rénové en 1988, c'est aujourd'hui un musée (classé patrimoine de la RAMI) qui présente sommairement l'histoire des gouverneurs mandchous de la Nouvelle ville, avec des statues de cire en costume d'époque.

Construite en 1706 au nord de la Vieille ville, la Résidence de la Princesse (Gongzhu fu) - une fille de l'empereur Kangxi donnée en mariage au petit-fils du Tüsijetü Qan des Qalq-a peu avant leur allégeance aux Mandchous en 1691 - a été occupée par une partie de l'École normale de Kökeqota en 1921, qui détruisit le jardin et rajouta des salles de classe. À la fin des années 1980, les bâtiments, en mauvais état mais encore occupés pour certains, étaient noyés dans des constructions nouvelles formant le sud de l'École normale. Puis celle-ci déménagea, et la résidence fut entièrement restaurée dans les années 1990, pour devenir le musée municipal d'Histoire, ouvert le $1^{\mathrm{er}}$ août 
2001. Le musée conserve des collections portant sur l'histoire de Kökeqota depuis le néolithique, y compris les peintures murales du Grand temple précédemment mentionnées. Il exposait en 2002 la collection de Yang Lu'an (calligraphe et collectionneur, qui a offert sa collection de peinture et de numismatique à Kökeqota en 1993). On visite encore en ville le mémorial à Ulanfu (1906-1988), Wulanfu jinianguan, édifié en 1992 dans le jardin botanique (Zhiwu yuan) sur le plan de celui de Gengis Khan, mais en plus grand $\left(2100 \mathrm{~m}^{2}\right)^{36}$. Il est devenu un site de tourisme-pèlerinage.

Pour attirer les touristes dans les nouveaux quartiers, on éparpille le patrimoine. Huit constructions typiques des architectures $d u \mathrm{XVI}^{\mathrm{e}}$ au $\mathrm{xIX}^{\mathrm{e}}$ - résidences, temples, scènes de théâtre et boutiques -, situées jadis dans la Vieille ville, ont été démontées brique par brique, pour être remontées dans le Jardin d'exposition d'architectures Ming et Qing (Ming Qing bolan yuan), situé dans le nouveau quartier de Jinchuan, à $5 \mathrm{~km}$ à l'ouest de la ville ${ }^{37}$.

Le musée de Mongolie-Intérieure, fondé en 1957 et détruit pendant la révolution culturelle, a été rebâti aussitôt après (FIG. 2) ${ }^{38}$. Il n'a pas été rénové depuis longtemps et semble bien poussiéreux. Il comporte quatre sections: flore et faune préhistoriques dont les célèbres dinosaures, objets archéologiques du néolithique aux Yuan, ethnographie de la "minorité" mongole et des autres minorités de la Région autonome, et périodes républicaine et révolutionnaire. La présentation historique insiste sur l'ancienneté de la présence han dans la région, alors que ce musée conserve dans ses réserves de nombreuses pièces postérieures aux Yuan qui n'ont jamais été exposées. Soulignons que les activités archéologiques relèvent essentiellement du sauvetage, telles les fouilles qui, à l'occasion de la construction de l'hôtel Bayantala, ont mis au jour quelques pièces bouddhiques (dont une belle statue de bodhisattva en cuivre doré).

Mais le touriste n'est pas incité à se contenter des sites intra-urbains. L'endroit à visiter pour qui ne passe qu'un jour à Kökeqota est la tombe de Wang Zhaojun (Zhaojun mu). Donnée en mariage au roi des Xiongnu en 33 av. J.-C., Wang Zhaojun symbolise le courage et le sacrifice exemplaire d'une princesse chinoise en échange de la paix. La promotion de ce site (qui ne présente qu'un monticule de terre surmonté d'un petit pavillon, une statue moderne du couple à cheval et un bas-relief monumental, à $9 \mathrm{~km}$ au sud de la ville) n'est pas innocente, puisqu'elle met en valeur l'ancienneté des (bonnes) relations sino-" barbares" ${ }^{39}$. Des représentations théâtrales "culturelles" relatant l'histoire de la malheureuse princesse s'y déroulent (" ce n'est pas une tombe, mais un mémorial historique de l'amitié inter-ethnique ", on parle même de la " culture Zhaojun " ${ }^{40}$ ).

Une autre destination touristique classique est la pagode blanche octogonale (Wanbu huayanjing ta) du temple Daming, datant de la fin du $x^{e}$ siècle - vestige de la ville de Fengzhou des Kitan (Liao) - à $20 \mathrm{~km}$ à l'est de Kökeqota. La pagode et le terrain alentour furent fouillés en 1960, 1976 et 1985, on y découvrit les plus anciens papiersmonnaies connus au monde. Les objets découverts sont conservés au musée de Mongolie-Intérieure et au musée d'Histoire de la Chine à Pékin. On a reconstruit le sommet de la pagode, qui a été classée monument protégé à l'échelon national en 1982.

En réalité, tous ces sites prestigieux ne sont que des amuse-gueules pour le touriste moyen. Ce qu'il veut voir, c'est la steppe, et les agences de voyage recrutent sans problème, abordant les touristes dans les couloirs de leur hôtel au doux son de caoyuan ("steppe"). On recherche le folklore mongol reconstitué, "domestiqué": jeux du 
nayadum, danses "traditionnelles", nourriture "typiquement mongole" (la fondue naturellement ${ }^{41}$, la soupe de tripes de mouton au petit déjeuner, le mouton bouilli à manger avec les doigts et le thé salé au lait), robes mongoles que l'on emprunte pour la photo - ce sont des déguisements dans un matériau synthétique léger, brillant et multicolore, censés être un lointain pastiche de la robe tümed. Un autre loisir des “ habitants de Mongolie-Intérieure " (Neimenggu ren) d'ethnie han consiste à se rendre dans la campagne pour profiter à outrance de l'hospitalité "sacrée" des Mongols. Les Mongols détestent leurs incursions, mais n'ont d'autre choix que de les recevoir (rappelons que l'hospitalité mongole est également un passage obligé des touristes occidentaux en Mongolie).

La destination classique vers la steppe est Sir-a mören (chin. Zhaohe, Xilamuren caoyuan), à $90 \mathrm{~km}$ au nord de Kökeqota, un camp de yourtes-hôtels posées sur du béton. On peut y voir un monastère filiale du Siregetü zuu, le Sir-a mören zuu, mais surtout on monte à cheval, à chameau ou dans des chariots à l'ancienne tirés par des bœufs, on assiste à des courses de chevaux et de chameaux, à des représentations de lutte, à des spectacles de chants mongols. On peut également s'inviter dans les yourtes alentour, où vivent des Mongols tenus de revêtir des costumes clinquants dès qu'ils voient un touriste. On peut aussi se rendre à Küiten sili (" plateau froid ", chin. Huitengxile), steppe plus " authentique ", à $135 \mathrm{~km}$ au nord-est de Kökeqota, ou à Gegen tal-a (Gegen tala caoyuan, à $150 \mathrm{~km}$ au nord, près de la résidence princière des Dörben keüked). On s'y promène dans des chariots surmontés d'une coupole-yourte, tirés par un chameau.

Depuis quelques années, des villages de vacances (avec yourtes, karaoké, billard, simulation de mariage mongol, parc d'attractions, etc.) poussent comme des champignons, non seulement dans la steppe, mais également en forêt, en bordure du fleuve Jaune, pour attirer les citadins (FIG. 12). Citons par exemple :

- le lac Qara usu (chin. Hasu hai, également appelé le "lac de l'Ouest à l'extérieur des passes " ${ }^{42}$ ), à $70 \mathrm{~km}$ à l'ouest de Kökeqota ;

- le jardin écologique des Cent Pavillons de la montagne méridionale de Helinge'er, à $45 \mathrm{~km} \mathrm{au}$ sud de Kökeqota (Nanshan shengtai baiting gongyuan), avec des reproductions de pavillons célèbres et des monnaies géantes de $2 \mathrm{~m}$ de haut ;

- la forêt nationale de Hadamen (Hadamen guojia senlin gongyuan), à $27 \mathrm{~km}$ au nord de Kökeqota, dans les montagnes ;

- la zone de développement touristique Üsütü (Wusutu lüyou kaifa qu), ouverte en 1992, qui comprend un lac, une portion de la muraille des Royaumes Combattants et le monastère Üsütü mentionné plus haut ;

- les plages du fleuve Jaune (Huanghe fengjing qu) et le jardin du lac méridional (Nanhu gongyuan) dans le district de Toytu (Tuoketuo);

- le réservoir d'eau de Wanjiazhai (Wanjiazhai shuiku, district de Qingshuihe);

- le lac et le barrage de Baieryesha (Baieryesha ba, district de Helinge'er, à $75 \mathrm{~km}$ au sud de Kökeqota), où l'on peut nager et voir un élevage d'autruches. Les autorités se vantent de cette réalisation dans un endroit encore désertique il y a vingt ans ;

- le jardin botanique de Nantianmen (Nantianmen zhiwu yuan, district de Helinge'er, à $65 \mathrm{~km}$ au sud de Kökeqota).

Plus proches de la ville et destinés aux citadins de Kökeqota qui y passent leurs weekends, on trouve :

- le village de vacances Xiangcun yuan (Xiangcun yuan dujia cun), à $7 \mathrm{~km}$ au sud-ouest de Kökeqota ; 
- le "hameau de montagne pour éviter la chaleur" ${ }^{43}$ des monts Daqing (Daqingshan bishushanzhuang), à $17 \mathrm{~km}$ au nord-ouest de Kökeqota; on peut y voir une portion reconstruite de la grande muraille des Royaumes Combattants, un temple de Guandi, les vestiges d'un “palais de voyage " d'un empereur des Wei du Nord et des yourtes.

Fig. 12 - Carte des principaux sites touristiques de la région de Kökeqota

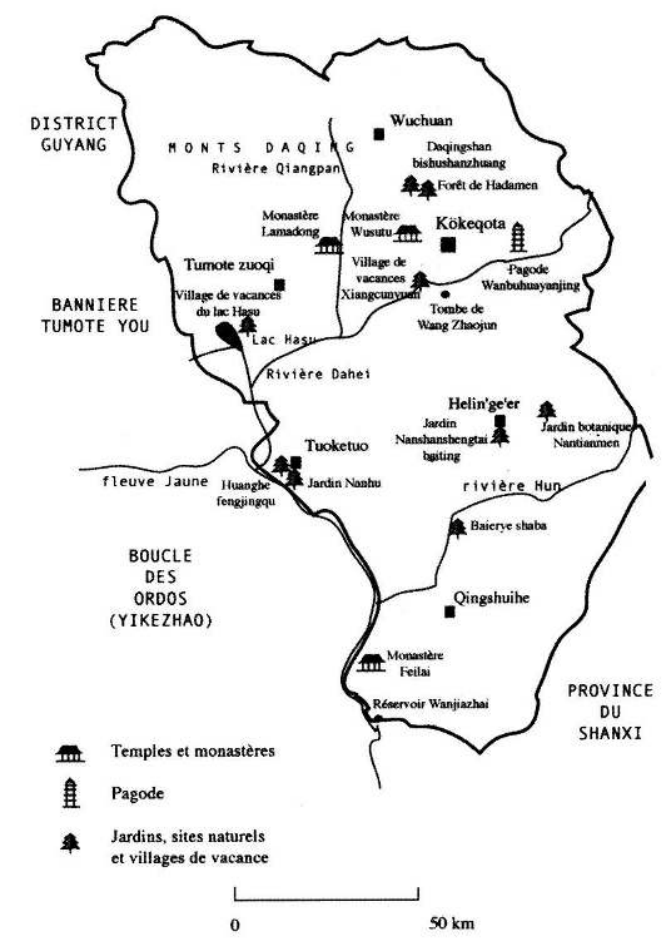

\section{Un patrimoine architectural en danger dans toute la Chine}

À Kökeqota, la sauvegarde du patrimoine entre en contradiction avec les exigences de l'urbanisme et de l'aménagement du territoire. Les moyens financiers et coercitifs manquent pour mettre en œuvre la politique de protection du patrimoine, notamment dans les sites urbains où la pression immobilière finit par l'emporter. La promotion du tourisme de masse - le tourisme individuel étant encore peu développé en Chine - est à double tranchant, puisqu'elle incite à la restauration de monuments mais implique l'aménagement des sites (construction de parkings pour les cars devant les monuments historiques et de grands restaurants, élargissement des rues, etc.), leur rentabilisation et la folklorisation des lieux de culte (boutiques, voire galeries marchandes, dans les monastères).

Kökeqota est loin d'être la seule ville de Chine à subir la destruction et la défiguration de son patrimoine. Par exemple, la démolition de l'habitat pékinois traditionnel (les hutong) est fortement accélérée par la préparation des Jeux Olympiques de 2008. Hormis l'architecture monumentale et de rares parcelles du tissu urbain ancien, la quasitotalité du vieux Pékin est sur le point de disparaître $-\mathrm{y}$ compris les 40 " quartiers historiques " protégés. Bien que la Chine se soit engagée auprès de l'Unesco à respecter 
les abords immédiats de la Cité Interdite, puisque l'ancien palais est inscrit sur la liste du patrimoine mondial, le quartier de Nanchizi vient d'être rasé ${ }^{44}$; parallèlement des petits temples rouvrent, les musées se multiplient. Des villages de Chine du Sud, au contraire, ont montré à quel point la restauration de vieux quartiers peut être profitable tant aux habitants qu'aux touristes.

Un autre exemple notable est celui de Lhasa, qui entre également dans le "programme de développement des régions de l'Ouest". Les autorités de Lhasa (d'après les statistiques officielles de 2001, 230000 habitants dont $70 \%$ de Chinois, sur $54 \mathrm{~km}^{2}$ ), qui préparent l'arrivée du chemin de fer dans la capitale de la région autonome du Tibet, prévoient de quadrupler la surface de la ville d'ici à 2015 et d'accroître la population de $30 \%$ dans les quatre prochaines années, avec une nouvelle zone de développement économique ${ }^{45}$. Contrairement à la Mongolie-Intérieure, le Tibet a un médiatique défenseur de sa culture sur la scène internationale, le dalaï-lama, et est soutenu par plusieurs organisations internationales pro-tibétaines. Rien n'a pu, pourtant, éviter les "travaux d'embellissement" de cette ville qui se veut une vitrine de la modernisation du Tibet. Bien que le Potala ait été inscrit sur la liste du Patrimoine mondial en 1995 par l'Unesco, on a ouvert devant lui une vaste place. Le cœur historique s'est réduit comme une peau de chagrin autour du Jokhang (inscrit en septembre 2000 sur la liste de l'Unesco). Ce processus a failli s'interrompre en 1998, mais a repris en 2002, avec de nouvelles destructions d'une ampleur sans précédent depuis la révolution culturelle. Même les maisons anciennes, restaurées par une association occidentale qui œuvrait depuis dix ans à la sauvegarde du vieux Lhasa et dont les membres ont été expulsés du Tibet en 2001, ont été rasées pour laisser place à des immeubles modernes, vaguement inspirés d'un style sino-tibétain. “Les autorités de Pékin ont avoué qu'elles étaient incapables de mettre un terme à la frénésie de spéculation des responsables locaux du Parti communiste, confirmant l'existence d'une administration coloniale qui n'en fait qu'à sa tête, dans la plus grande impunité nationale et internationale" " ${ }^{46}$. L'UNESCO, qui est pourtant parfaitement au courant de ces destructions, semble fermer les yeux tant pour Pékin que pour Lhasa.

\section{Kökeqota, ville chinoise?}

62 Le patrimoine architectural proprement mongol de Mongolie-Intérieure - essentiellement les monastères - a mieux survécu que le patrimoine chinois et, aujourd'hui, ce sont des habitations et boutiques essentiellement occupées par des Han que l'on rase. D'un autre côté, les sites les plus promus, comme la tombe de Wang Zhaojun, tendent à mettre en valeur l'histoire han de Kökeqota, " un des lieux de naissance de la nation chinoise" depuis l'âge de la Pierre ${ }^{47}$. La majorité des édifices classés n'est pas mongole, ce qui voudrait faire accroire que ces nomades, installés seulement "récemment" dans la région, héritent d'un riche passé ${ }^{48}$. Les Han de Mongolie-Intérieure prétendent être plus indigènes que les Mongols : ils se seraient installés dans la région il y a plus de deux mille ans. Dans les années 1990, des intellectuels han ont mis à contribution l'histoire et l'archéologie pour étayer cette revendication qui permet de légitimer la colonisation han de zones jadis réservées aux minorités ${ }^{49}$. Nous avons également vu comment les Chinois apposent leur sceau sur le patrimoine mongol par des restaurations abusives. 
63 La "ville féodale décadente" sino-mongole est pleinement devenue une "nouvelle capitale socialiste chinoise" jouant un rôle de phare dans le programme de développement de l'Ouest. En apparence, Kökeqota est aujourd'hui une ville principalement chinoise, même si les enseignes arborent des panneaux dans les deux langues. Seuls quelques monastères rappellent encore son prestigieux passé mongol, et pourtant Kökeqota reste la capitale intellectuelle des quatre millions de Mongols de Mongolie-Intérieure, puisque y sont concentrés les principales universités, les archives de Mongolie-Intérieure, les bibliothèques et le plus grand musée de la "Région autonome".

\section{BIBLIOGRAPHIE}

Alexander, André et Pimpim de Azevedo

1998 The Old City of Lhasa. Report from a Conservation Project [avec un relevé du plan de la ville]

(Berlin, Tibet Heritage Fund/Verlag Freie Kultur Aktion).

Barnett, Robert

2003 A City, Its Visitors, and the Odour of Development, in Françoise Pommaret (éd.), Lhasa in the Seventeenth Century. The Capital of the Dalai Lamas (Leiden/Boston, Brill), pp. 199-226.

Blondeau, Anne-Marie et Katia Buffetrille (éd.)

2002 Le Tibet est-il chinois ? Réponses à cent questions chinoises (Paris, Albin Michel).

Bulag, Uradyn E.

1996 Nationalism and Hybridity (Oxford, Clarendon Press).

1998 The Cult of Ulanhu in Inner Mongolia. History, Memory, and the Making of National Heroes, Central Asian Survey,XVII, 1, pp. 11-33.

Charleux, Isabelle

1998 Histoire et architecture des temples et monastères lamaïques de Mongolie méridionale, thèse de doctorat de l'Université de Paris IV-Sorbonne, non publiée.

2003 Le Patrimoine bâti dans la région “ autonome " chinoise de Mongolie-Intérieure : état actuel et avenir des architectures bouddhiques, in Maria Gravari-Barbas et Sylvie Guichard-Anguis (éd.), Regards croisés sur le patrimoine dans le monde à l'aube du XXI siècle (Paris, Presses de l'Université de Paris-Sorbonne), pp. 223-242.

2004 The Reconstruction of Buddhist Monasteries in the Chinese Autonomous Region of Inner Mongolia. Between Sanctuary and Museum, in Agata Bareja-Starzynska et Hanna Havnevik (éd.), Revival of Buddhism in Mongolia after 1990 (Varsovie), à paraître.

Chen, Xiejun et al. (éd.)

2000 Caoyuan guibao. Nei Menggu wenwu kaogu jingpin [Treasures on Grassland. Archaeological Finds from the Inner Mongolia Autonomous Region] (Shanghai, musée de Shanghai).

Chenivesse, Sandrine

2002 L'avenir du vieux Pékin et le conflit entre modernisation et conservation, Bulletin de l'École Française d'Extrême-Orient, 89, pp. 364-374. 
Edelmann, Frédéric

2002 De l'ancien, Pékin fait table rase, Le Monde, samedi 19 octobre 2002, p. 31.

Elverskog, Johan

2003 The Jewel Translucent Sûtra. Altan Khan and the Mongols in the Sixteenth Century (Leiden/

Boston, Brill) [Brill's Inner Asian Library, 8].

Fresnais, Jocelyne

2001 La Protection du patrimoine en République populaire de Chine 1949-1999 (Paris, Editions du CTHS).

Gaubatz, Piper Rae

1996 Beyond the Great Wall. Urban Form and Transformation on the Chinese Frontiers (Stanford,

Stanford University Press).

Halde, Jean-Baptiste du (SJ)

1736 Description géographique, historique, chronologique, politique et physique de l'Empire de la Chine et de la Tartarie (La Haye, Henri Scheurleer), 4 vol.

Huang, Lisheng

1982 You junshi zhenglüe dao chengshi maoyi : Nei Menggu Guisui diqu de shehui jingji bianqian (14 shiji zhong zhi 20 shiji chu) [Des guerres et des pillages aux villes et au commerce : les changements dans l'économie et la société de la région de Guisui en Mongolie-Intérieure (du milieu du $\mathrm{xIV}^{\mathrm{e}}$ siècle au début $\mathrm{du} \mathrm{xx}^{\mathrm{e}}$ )] (Taipei, Guoli Taiwan shifan daxue lishi yanjiu suo).

Huc, Régis Évariste

[1924] 1987 Souvenirs d'un voyage dans la Tartarie et le Thibet pendant les années 1844, 1845 et 1846, édition revue et commentée par le père J. M. Planchet (Paris, L'Astrolabe-Peuples du Monde), 2 vol.

Huhehaote lüyou [Huhhot Tourism]

2001 (Kökeqota, Nei Menggu renmin chubanshe).

Huhehaote lüyou lansheng [Glance over Huhhot Tourism]

2002 (Kökeqota, Nei Menggu renmin chubanshe).

Hyer, Paul

1982 An Historical Sketch of Köke-Khota, City Capital of Inner Mongolia, Central Asiatic Journal, XXVI, 1-2, pp. 56-77.

Inner Mongolia

2001 (Pékin, China Intercontinental Press) [West China].

Jankowiak, William

1993 Sex, Death and Hierarchy in a Chinese City. An Anthropological Account (New York, New York University Press).

Kessler, Adam T. et al.

1993 Empires Beyond the Great Wall. The Heritage of Genghis Khan (Los Angeles, Natural History Museum of Los Angeles County).

Larsen, Knud et Amund Sinding-Larsen

2001 The Lhasa Atlas. Traditional Tibetan Architecture and Townscape (London, Serindia).

Mackerras, Colin

1994 Religion, Politics and the Economy in Inner Mongolia and Ningxia, in H. Kaplan et Donald W. Whisenhunt (éd.), Opuscula Altaica. Essays presented in Honour of Henry Schwarz (Bellingham, Western Washington University, Centre for East Asian Studies), pp. 437-464. 
Nei Menggu zizhi qu wenwu baohu guanli weiyuanhui [Comité de gestion de la protection du patrimoine de la région autonome de Mongolie-Intérieure]

1988 Nei Menggu zizhi qu zhongdian wenwu baohu danwei jieshao [Présentation des sites protégés du patrimoine culturel de la région autonome de Mongolie-Intérieure] (Kökeqota, Nei Menggu renmin chubanshe).

Ribes, Jean-Paul

2002 Dépêche de l'Agence France-Presse (26 juin 2002), cité dans Tibet Information Network du 28 juin 2002.

Sun, Lizhong

1997 Huhehaote wenwu [Patrimoine de Kökeqota] (Kökeqota, Nei Menggu renmin chubanshe).

Tibet Heritage Fund

19991998 Annual Report (Hong Kong).

Tibet Information Network

13 juin 2001

Yi, Gu et Gao Geng'en

[1908a] 1968a Suiyuan quan zhi [Monographie complète [de la région de] Suiyuan] (Taipei, Chengwen chubanshe) [Zhongguo fangzhi congshu, Saibei difang, 9].

[1908b] 1968b Tumote zhi,encore appelé Tumote qi zhi [Monographie des Tümed/de la bannière des Tümed] (Taipei, Chengwen chubanshe) [Zhongguo fangzhi congshu, Saibei difang, 16].

Zhamusu

1997 Wo de huofo shengya [Ma vie], entrevue transcrite par Wuyun Gaowa, in Yang Jizeng (éd.), Nei Menggu lamajiao jili [Chroniques du lamaïsme en Mongolie-Intérieure] (Kökeqota, Nei Menggu wenshi shudian), pp. 258-264 [Nei Menggu wenshi ziliao, 45].

Zhang, Dingyi

1920 Suisheng [Monographie [de la région de] Suiyuan] (Shanghai, Taidong tushuju), 2 vol.

Zhang, Liang

2003 La naissance du concept de patrimoine en Chine. XIX ${ }^{e}-\mathrm{XX}$ siècles (Paris, Éditions Recherches/

IPRAUS) [coll. Archithèse].

Zhao, Chunping et a.l

1987 Nei Menggu lüyou [Guide de tourisme en Mongolie-Intérieure] (Kökeqota, Nei Menggu renmin chubanshe).

Zheng, Yufu et Zheng Zhichang

[1934] 1968 Guisui xian zhi [Monographie de Gui[hua] Sui[yuan]] (Taipei, Chengwen chubanshe, Zhongguo fangzhi congshu, Saibei difang, 10).

\section{ANNEXES}

\section{Glossaire}

Baieryesha ba 白二爺沙壩

Bailing miao 百霓廟

Caishen 財神

caoyuan草原 
Caoyuan guibao. Nei Menggu wenwu kaogu jingpin草原瑰寶-内蒙古文物考古精品

Chen Xiejun 陳焞君

Chenghuang miao 城隍廟

Da bansheng 大板升

Daming si 大明寺

Danan jie 大南街

Daqingshan bishushanzhuang 大青山避暑山莊

Dashengkui 大盛魁

Dazhao qianjie 大召前街

Dazhao (si) 大召 (寺)

fengmao風貌

Fengzhou 豐州

Gao Geng'en 高㿎恩

Gegen tala caoyuan 格根塔拉草原

Gongzhu fu 公主府

gutou骨頭

Guandi 關帝

Guihua cheng 歸化城

Guisui xian zhi 歸綏縣志

Hadamen guojia senlin gongyuan 哈達門國家森林公園

Hasu hai 哈素海

Helinge'er 和林格爾

Huang Lisheng 黃麗生

Huhehaote 呼和浩特

Huhehaote lüyou 呼和浩特旅游

Huhehaote lüyou lansheng呼和浩特旅游筧勝

Huhehaote wenwu 呼和浩特文物

hutong胡同

Huzhai cun aobao 忽寨村敖包

Huanghe fengjing qu 黃河風景區

Huiminqu 回民區

Huitengxile caoyuan 輝騰席勒草原

huoguo火鍋

Jiangjun yashu 將軍衙暑

Jinchuan 金川

Jiucheng 舊城

Jiucheng beimen qichezhan 舊城北門汽車站

Jushilin居士林

Lamadong si 喇嘛洞寺

lamian拉麵

Liulichang 琉璃廠

Maixiang cun 麥香村

Ming Qing bolan yuan 明清博覽園

Naimuqi zhao 乃莫齊召

Nanchizi 南池子

Nanhu gongyuan 南湖公園

Nanshan shengtai baiting gongyuan 南山生態百亭公園

Nantianmen zhiwu yuan 南天門植物園 
Nei Menggu bowuguan 内蒙古博物館

Nei Menggu lamajiao jili 内蒙古喇啝教紀例Nei Menggu lüyou 内蒙古旅游Nei Menggu

saima chang 内蒙古賽馬場

Neimenggu ren 内蒙古人

Nei Menggu zizhi qu wenwu baohu guanli weiyuanhui 内蒙古自治區保護管理委員會

Nei Menggu zizhi qu zhongdian wenwu baohu danwei jieshao内蒙古自治區重點文物保護單

位介紹

Puhui si 普會寺

Qiaoerqi zhao 巧爾气召

Qingshuihe 清水河

Renmin gongyuan 人民公園

shaodaomian稍刀麵

shaomai稍麥

Shengle 盛樂

shuanyangrou涮羊肉

Siziwang 四子王

Suisheng 綏乘

Suiyuan 綏遠

Suiyuan quan zhi 綏遠全志

Sun Lizhong 孫利中

Tongshun jie 通順街

Tongyi shanshe統一善社

Tumote qi zhi 土默特旗志Tumote xuexiao 土默特學校

Tumote zhi 土默特志Tuoketuo 托克托

Wanbu huayanjing ta 萬部華嚴經塔

Wanjiazhai shuiku 萬家寨水庫

Wang Zhaojun 王昭君

Wencheng (Page number)文成

Wenmiao 文廟

Wo de huofo shengya 我的活佛生涯

Wugongba aobao 蜈蚣壩敖包

Wulanfu jinianguan 烏蘭夫紀念館

Wusutu lüyou kaifa qu烏素圖旅游開發區

Wusutu zhao 鳥素圖召

Wuta si 五塔寺

Wuyun Gaowa 烏雲高娃

Xilamuren caoyuan 席拉穆仁草原

Xilitu zhao 席力圖召

Xiangcun yuan dujia cun 香村園度假村

Xiaozhao 小召

Xincheng 新城

Xinhua guangchang 新華廣場

Yang Jizeng 楊繼曾

Yang Lu'an 楊魯安

$\mathrm{Yi} \mathrm{Gu}$ 貽撀

Yishiting議事廳

You junshi zhenglüe dao chengshi maoyi : Nei Menggu Guisui diqu de shehui jingji bianqian (14

shiji zhong zhi 20 shiji chu) 由軍事征掠到城市貿易: 内蒙古歸綏地區的社會經濟變遷(14 
世紀中至20世紀初)

Yunzhong 雲中

Zhang Dingyi 張鼎彝Zhamusu 扎木蘇

Zhaocheng 召城

Zhao Chunping 趙春萍

Zhaohe 召河

Zheng Yufu 鄭裕学

Zheng Zhichang 鄭植昌Zhaojun mu 昭君墓

Zhiwu yuan 植物園

Zhongshan jie 中山街

\section{NOTES}

1. "Huhehaote" en pinyin; "Hohhot" suivant un système chinois de romanisation des termes mongols, employé entre autres dans les cartes géographiques.

2. Les sites protégés sont classés en six catégories : sites révolutionnaires et mémoriaux, sites rupestres, architectures anciennes et mémoriaux historiques, inscriptions lapidaires et gravures, sites archéologiques, tombes historiques.

3. Voir Hyer (1982, pp. 56-61).

4. Le terme qota, qui désigne dans le vocabulaire de l'élevage l'enclos à bétail, prend par extension le sens de forteresse et de ville, généralement murée. Kökeqota est encore appelée au $\mathrm{XVI}^{\mathrm{e}}$ siècle Jeke baising, "le grand baising”, mot qui vient probablement du chinois et qui désignait alors les bâtiments en dur et les villages que bâtissaient les Chinois agriculteurs installés en Mongolie méridionale. Baising signifie aujourd'hui en mongol "bâtiment", " maison", et a perdu son sens de "village". L'histoire de Kökeqota est fondée sur les sources suivantes : zhangDingyi (1920), zheng Yufu et zheng Zhichang ([1934] 1968), YI Gu et Gao Geng'en ([1908a] $1968 a$ et $[1908 b] 1968 b)$ et Elverskog (2003). Pour plus de détails, cf. Hyer (1982), pp. 56-77), Huang Lisheng (1995) et charleux (1998, pp. 58-68 et 130-133).

5. Siège du gouvernement.

6. Le palais est transformé en yamen du gouverneur de Kökeqota lorsque, en 1640, les Mandchous éliminent la maison princière des Tümed, accusée de trahison, et lui substituent leur propre organisation.

7. L'empereur Guan : Guan Yu, dieu martial chinois dont le culte a été diffusé en Mongolie par les Mandchous.

8. Une des plus anciennes descriptions de la ville est celle du père Gerbillon, voyageant dans la suite de l'empereur Kangxi, en 1687-1688 : “ C'est une petite ville qu'on dit avoir été autrefois fort marchande, et d'un grand abord, pendant que les Tartares de l'Oüest étoient les maîtres de la Chine : à présent c'est fort peu de chose : les murailles bâties en briques sont assez entières par dehors, mais il n'y a plus de rempart au dedans : il n'y a même rien de remarquable dans la ville, que des Pagodes et des Lamas. On en voit plusieurs bien bâtis, plus beaux et plus ornez que la plûpart de ceux que j'ai vûs à la Chine. Presque toutes les autres maisons ne sont que des huttes de terre (du Halde 1736, vol. IV, pp. 122-123). Les remarques du père Huc, qui y séjourne en 1844, sont semblables (Huc [1924] 1987, vol. I, p. 194).

9. Ces yourtes et maisons rondes n'ont pas subsisté (Gaubatz 1996, p. 246).

10. Pour le développement de Kökeqota au Xx siècle, cf. Gaubatz (1996, pp. 281-289, 293-294 et 302-304).

11. Pour une ethnographie moderne de Kökeqota, cf. Jankowiak (1993). Gaubatz (1996, p. 343 note 2) discute les chiffres donnés par Jankowiak (1993, pp. 10 et 16). 
12. Ce parc fut fondé en 1931 sous le nom de parc de la Source du dragon. Le gouvernement communiste l'a rénové en 1950 et a changé son nom en parc du Peuple.

13. Tongyi shanshe, sanctuaire et lieu de réunion d'un groupe religieux laïc pratiquant l'écriture inspirée (dans le cadre d'un culte médiumnique) et faisant des œuvres de bienfaisance. Ce type de sanctuaire était très répandu à l'époque républicaine.

14. Il ne reste aujourd'hui de l'ancienne Suiyuan que le yamen du général (voir plus loin) et une petite portion du mur d'enceinte.

15. Le terme de Ville nouvelle ne désigne plus seulement Suiyuan, mais s'étend à toute la ville, excepté la Vieille ville.

16. Voir également le célèbre "mausolée" de Gengis Khan dans les Ordos fait de trois constructions octogonales coiffées chacune d'un dôme-yourte bordé d'auvents chinois (1956).

17. Au moment de la construction du musée, le cheval fut orienté vers le nord, en direction de la Mongolie. Après la révolution culturelle, l'édifice ayant été partiellement détruit fut reconstruit avec une nouvelle statue de cheval faisant cette fois face au sud, pour " ramener la minorité mongole vers le centre du territoire chinois" (d'après Bulag 1996, p. 177).

18. Fresnais (2001, pp. 101, 125-127, 264).

19. Témoignage de Jin Shen, chercheur chinois envoyé en Mongolie-Intérieure pendant la révolution culturelle.

20. Sur la politique patrimoniale en Mongolie-Intérieure, cf. Charleux (2003); en Chine, cf. Fresnais (2001) et Zhang Liang (2003). La promulgation d'une première liste de bâtiments protégés en 1961 par le Bureau de la gestion du patrimoine n'avait pas empêché les destructions de la révolution culturelle. Chaque année, la liste des sites protégés s'allonge, incluant en Mongolie-Intérieure des vestiges de villes, murailles, bâtiments, tombes, etc., des sites révolutionnaires, ainsi que des monuments récents et symboliques comme le "mausolée de Gengis Khan ". Il s'agit avant tout d'une liste sur le papier, qui permet aux autorités de MongolieIntérieure de se vanter des quelque "vingt mille objets culturels préservés".

21. Dazhao, du chinois da, "grand", et zhao, transcription phonétique du mongol zuu, "temple", traduit donc Jeke zuu. Cependant on rajoute aujourd'hui en chinois si, " monastère ”, car le sens de zhao/zuu a été oublié : Dazhao est pris comme un nom propre.

22. Sur le renouveau du bouddhisme en Mongolie-Intérieure, cf. Charleux (2004 // à paraître).

23. C'est un Tibétain, marié (selon une tradition ancienne propre aux Siregetü qutuytu, pourtant d'obédience guélougpa) avec enfants, enthousiaste de la politique de Deng Xiaoping. Voir son autobiographie (Zhamusu 1997) et son interview de 1992 par Mackerras (1994, pp. 450, 452 et 458).

24. Branche de l'Association bouddhique de la RAMI, dépendant du bureau des Affaires religieuses et incluant des membres du Parti. Une des tâches principales du bureau des Affaires religieuses est de négocier le départ de l'entreprise ou des particuliers installés dans un temple réaffecté au culte et de les reloger.

25. Les gouvernements locaux et la RAMI ont versé depuis 1984 cinq millions de yuans d'indemnités au clergé.

26. Dans la Chine impériale, la troisième religion n'est pas le bouddhisme tibétain, mais le confucianisme.

27. Montjoie, demeure des divinités locales.

28. Pour les critères définissant un "secteur historique", cf. Fresnais (2001, p. 195) et Zhang Liang (2003, pp. 209-218).

29. Sun Lizhong (1997, pp. 83-80) décrit l'animation du quartier.

30. Gaubatz (1996, pp. 234 et 245-246).

31. Unephotographie ancienne de larue avant du Grand temple publiée dans un guide touristique (Huhehaote lüyou lansheng 2002, p. 20) fait la promotion de cette rue, pourtant déjà rasée lors de la publication de l'ouvrage en 2002 ! 
32. Sur le projet de Liulichang et le concept de fengmao (" allure", "style", " caractère", "identité ") comme critère principal dans la restauration des "Villes célèbres d'histoire et de culture ", cf. Zhang Liang (2003, pp. 158-162 et 179-188).

33. Ce programme à double tranchant se traduit avant tout par une surexploitation des matières premières: l'Ouest pauvre doit approvisionner les riches régions côtières. Au programme également, des fondations de villes nouvelles, une amélioration générale des infrastructures, la préservation de l'environnement, la gestion des ressources en eau, des progrès en matière d'éducation, et un partenariat commercial privilégié avec la Mongolie. Voir Inner Mongolia (2001) : ouvrage de propagande présentant le développement actuel et futur de la région.

34. Inner Mongolia (2001, pp. 64-66).

35. Huhehaote lüyou lansheng (2002) et Huhehaote lüyou (2001). Un des premiers guides touristiques de Mongolie-Intérieure était Zhao Chunping et al. (1987).

36. L'État lui avait refusé un mausolée après sa mort - ce qui avait soulevé la protestation des Mongols (Bulag 1998).

37. Sun (1997) décrit plusieurs petits édifices préservés et classés patrimoines historiques : un bâtiment de l'ancien yamen (Grande salle de débats : Yishiting) de l'aile gauche des Tümed (1735), une entreprise de transport commercial mongol (Dashengkui), etc. Il est possible que ces bâtiments, ainsi que des boutiques de la " rue avant du Grand temple ", aient été déménagés dans ce musée.

38. Bulag (1998, p. 177).

39. De même, au Jokhang de Lhasa est glorifiée la princesse Wencheng, épouse chinoise du roi Songtsen Gampo [Srong btsan sgam po] .

40. Huhehaote lüyou lansheng (2002, p. 6).

41. Rappelons que ce célèbre plat (huoguo ou shuanyangrou), supposé avoir été inventé par un cuisinier de l'empereur Qubilai avant une bataille, est dit être chinois par les Mongols, et mongol par les Chinois.

42. Par comparaison avec le célèbre lac de l'Ouest de Hangzhou, à l'intérieur des passes.

43. Nom de la résidence des empereurs mandchous à Jehol (Chengde), au nord-est de Pékin. De même que le lac de l'Ouest à l'extérieur des passes, ces nouveaux toponymes sont parlants pour les touristes et cherchent à démontrer que les sites de Mongolie-Intérieure n'ont rien à envier à ceux de la Chine des dix-huit provinces.

44. Edelmann(2002, p. 31) et Chenivesse (2002).

45. Tibet Information Network du 13 juin 2001.

46. Ribes (2002). Sur la destruction de Lhasa, cf. Alexanderet DE Azevedo (1998), Blondeau et Buffetrille (éd.) (2002, pp. 271-275 et 417), Tibet Heritage Fund (1999), Larsen et Sinding-Larsen (2001) et Barnett (2003).

47. Traduit de Huhehaote lüyou (2001, pp. 1-2).

48. C'est également le message véhiculé par les grandes expositions du patrimoine archéologique des " peuples nomades de la steppe " présentant des objets du musée de MongolieIntérieure à Kökeqota, notamment en 1993-1995 "Genghis Khan. Treasures from Inner Mongolia" (Kessleret al. 1993) et en 2000 "Caoyuan guibao" (Chen Xiejun et al. 2000). Voir également Nei Menggu zizhi qu wenwu baohu guanli weiyuanhui (1988).

49. Un leader du Parti, d'“ ethnie " Han, donna à ce sujet une conférence en 1993 à l'École du Parti de Mongolie-Intérieure, qui fut très mal reçue. Il dut se rétracter devant les fortes protestations. 


\section{RÉSUMÉS}

Kökeqota, la «Ville bleue », capitale de la région autonome de Mongolie-Intérieure en Chine, conservait encore dans les années 1990 un patrimoine architectural d'une grande richesse. C'est ainsi qu'elle mérita en 1987 son classement parmi les «villes célèbres historiques et culturelles de niveau national » de Chine. Mais face à une forte expansion démographique, économique et urbanistique, le patrimoine architectural, même "protégé ", subit des dégradations irréversibles: la vieille ville, hormis quelques temples, est en train d'être rasée. Après une présentation de l'histoire de la ville, des transformations de la période communiste, de la politique patrimoniale et en particulier de la restauration du patrimoine religieux, l'article propose un état des lieux en 2002, évaluant l'ampleur des destructions et tentant de comprendre les enjeux actuels.

Kökeqota, the "Blue City", capital of the Inner Mongolia Autonomous Region in China, had preserved an architectural heritage of great richness until the 1990s. In 1987 it merited inclusion among the "nationally famous historic and cultural cities" of China. Yet, faced with strong demographic, economic, and urban expansion, the architectural heritage, although "protected", suffers irreversible degradation. The old city, except for a few temples, is being razed. After a presentation of the city's history, of its transformations during the Communist period, of the heritage policy, and in particular of the restoration of the religious structures, the article considers their state in 2002, evaluating the extent of the destruction and seeking to understand what is currently at stake.

\section{INDEX}

nomsmotscles Chahar, Gorlos, Horchin, Ordos, Tümet, Üzemchin

Mots-clés : anthropologie urbaine, développement urbain, patrimoine, renouveau du bouddhisme, restauration, tourisme

Thèmes : architecture, bouddhisme, changement, histoire, identité, minorités ethniques

Keywords : Buddhist revival, heritage, restoration, tourisme, urban anthropology, urban development

Index géographique : Chine, Mongolie Intérieure

\section{AUTEUR}

\section{ISABELLE CHARLEUX}

Isabelle Charleux est chargée de recherche au C.N.R.S. (Groupe de sociologie des religions et de la laïcité). Elle s'intéresse à la culture matérielle des Mongols, à l'art et à l'architecture du bouddhisme tibéto-mongol en Chine 